\title{
A computational model of mechanisms controlling experience-dependent reorganization of representational maps in auditory cortex
}

\author{
EDUARDO MERCADO III, CATHERINE E. MYERS, and MARK A. GLUCK \\ Rutgers University, Newark, New Jersey
}

\begin{abstract}
Cortical representations of sound can be modified by repeatedly pairing presentation of a pure tone with electrical stimulation of neuromodulatory neurons located in the basal forebrain (Bakin \& Weinberger, 1996; Kilgard \& Merzenich, 1998a). We developed a computational model to investigate the possible effects of basal forebrain modulation on map reorganization in the auditory cortex. The model is a self-organizing map with acoustic response characteristics mimicking those observed in the mammalian auditory cortex. We simulated the effects of basal forebrain modulation, using parameters intrinsic to the self-organizing map, such as the learning rate (controlling the adaptability of map nodes) and the neighborhood function (controlling the excitability of map nodes). Previous research has suggested that both parameters can be useful for characterizing the effects of neuromodulation on plasticity (Kohonen, 1993; Myers et al., 1996; Myers, Ermita, Hasselmo, \& Gluck, 1998). The model successfully accounts for experimentally observed effects of pairing basal forebrain stimulation with the presentation of a single tone, but not of two tones, suggesting that auditory cortical plasticity is constrained in ways not accounted for by current theories. Despite this limitation, the model provides a useful framework for describing experience-induced changes in auditory representations and for relating such changes to variations in the excitability and adaptability of cortical neurons produced by neuromodulation.
\end{abstract}

The dynamics of cortical processing are mediated by neuromodulatory mechanisms. Neuromodulatory chemicals are known to affect the sensitivities of cortical neurons to incoming signals, the modifiability of synaptic connections between neurons, and the oscillatory properties of cortical networks (for a review, see Hasselmo, 1995). How such effects are related to forming, maintaining, retrieving, or adapting neural representations is poorly understood. Computational models of cortical processing provide a useful theoretical framework for examining the roles neuromodulators play in the representation and transformation of sensory information (for reviews, see deCharms \& Zador, 2000; Fellous \& Linster, 1998; Gluck \& Myers, 2001).

Past computational models of processing in sensory cortices have often focused on replicating network dynamics and single-cell response properties. Many models have been proposed for describing how cortical sensitivities change during development and after injuries to receptors (see, e.g., Erwin, Obermayer, \& Schulten, 1995; Grajski \& Merzenich, 1990; Sirosh \& Miikkulainen, 1997;

This work was supported by an APA/NIMH postdoctoral fellowship and by the Rutgers-Newark MBRS program. The authors thank two anonymous reviewers of the manuscript for their insightful comments and suggestions. Correspondence concerning this article should be addressed to E. Mercado, Center for Molecular and Behavioral Neuroscience, Rutgers University, 197 University Ave., Newark, NJ 07102 (e-mail: mercado@pavlov.rutgers.edu).
Sutton, Reggia, Armentrout, \& D’ Autrechy, 1994; Swindale, 1996; Swindale \& Bauer, 1998; Tanaka, 1990). Less attention has been given to modeling changes in sensory representations induced by experience (Armony, ServanSchreiber, Cohen, \& LeDoux, 1995; Barkai, Bergman, Horowitz, \& Hasselmo, 1994; Benukskova, Diamond, \& Ebner, 1994; Elliott \& Shadbolt, 1998; Joublin, Spengler, Wacquant, \& Dinse, 1996).

Although experience-dependent changes in the response properties of sensory cortical neurons have been extensively investigated (for recent reviews, see Buonomano \& Merzenich, 1998; Edeline, 1999; Kaas, 1997), the mechanisms underlying cortical plasticity in adults remain unclear. The most detailed theoretical model of learning-induced plasticity developed to date describes how associative conditioning can change spectral sensitivities in the auditory cortex (Weinberger et al., 1990a; Weinberger et al., 1990b; Weinberger \& Bakin, 1998). This model specifies neuromodulatory actions that control how and when cortical representations will become reorganized.

Qualitative models of neuromodulatory effects, in combination with experimental data collected to test the predictions of these models, have led to significant progress toward understanding auditory cortical plasticity. These models are limited, however, in terms of how precisely they can predict the effects that particular experimental treatments will have on cortical representations of sound. More quantitative theoretical frameworks are needed in 
order to rigorously test the validity of current theoretical proposals concerning the role that neuromodulators play in cortical plasticity. Previous simulations of experiencedependent changes in auditory cortical processing (e.g., Armony et al., 1995) have not explicitly considered how neuromodulatory systems and preexisting topographical cortical sensitivities might affect the reorganization of auditory representations. Such variables can critically affect cortical reorganization (M. E. Diamond, Petersen, \& Harris, 1999; Dykes, 1997).

In this paper, we describe a connectionist model of auditory cortical processing that can be used to quantitatively describe the effects of neuromodulation on experiencedependent cortical plasticity. We constrained the organization and sensitivities of a neural network so that they paralleled properties seen in the mammalian auditory cortex and modeled the effects of modulatory processes on cortical reorganization, using parameters intrinsic to the learning algorithm used by the neural network. We investigated how variations in parameter values affected experience-induced changes in the responses of the neural network and compared these changes with recent electro- physiological descriptions of reorganization in the auditory cortex induced by pairing electrical stimulation of the basal forebrain with the presentation of tones.

Our goal was to develop a simple computational model of auditory cortical processing that could provide a precise theoretical framework for (1) investigating the roles of neuromodulation in experience-dependent reorganization of auditory representations and (2) testing the accuracy and sufficiency of more qualitative models of experience-induced plasticity in the auditory cortex. Preliminary reports of some of these data appeared in abstract form (Mercado, Myers, \& Gluck, 1999a, 1999b).

\section{A COMPUTATIONAL MODEL OF AUDITORY CORTICAL PROCESSING}

Numerous cortical models of varying computational complexity and physiological realism have been developed (see, e.g., Abbott \& Sejnowski, 1999; Arbib, 1998; Bower \& Beeman, 1998). Two general classes of auditory cortex models are prevalent: signal-processing models and neural network models (see Figure 1). Signal-processing
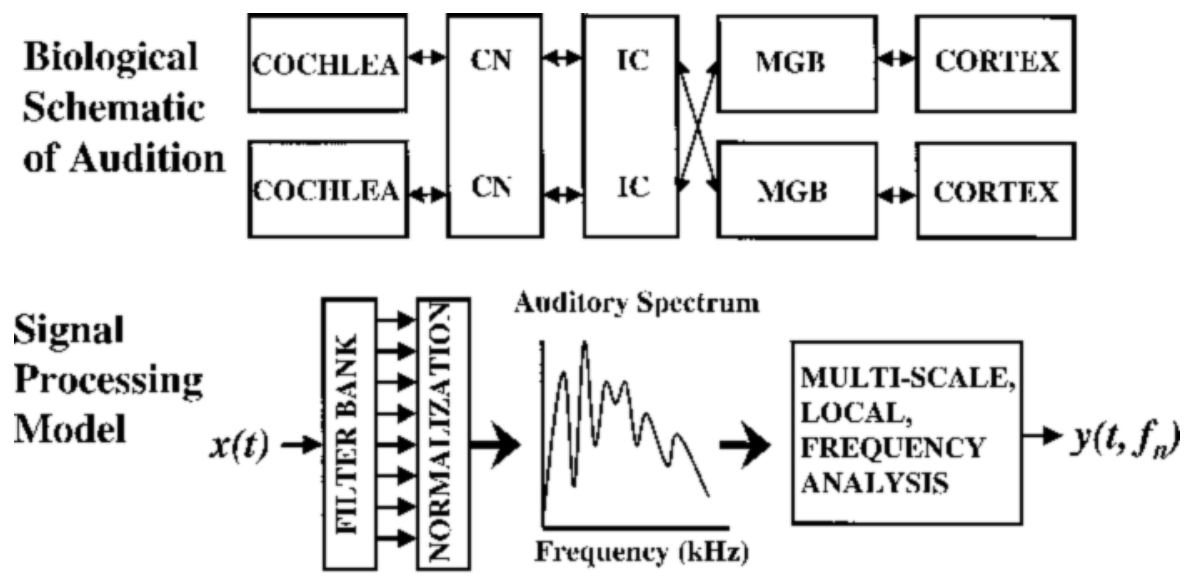

Neural
Network
Model

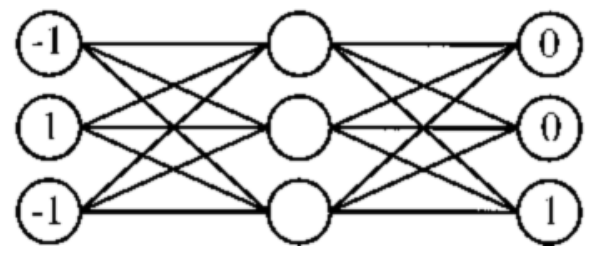

Figure 1. Models of audition. CN, cochlear nucleus; IC, inferior colliculus; MGB, medial geniculate body. The biological model involves a multimodule loop composed of converging and diverging neural connections. Signal-processing models typically involve sequential transformations of a one-dimensional waveform, $x(t)$, measuring variations in pressure over time. First, $x(t)$ is transformed by a set of bandpass filters simulating cochlear processing. Then, these signals are normalized, simulating processing by hair cells and auditory ganglia, to generate an auditory spectrum. Finally, the auditory spectrum is transformed into an $\boldsymbol{n}$-dimensional space, $y\left(t, f_{n}\right)$, that describes features of the sound, such as pitch and signal bandwidth. Neural network models are typically much more abstract. Inputs are often represented as binary vectors that code measured properties of sounds, and outputs often reflect activity levels of nodes. Transitions between stages (or layers) involve matrix transformations. Signal-processing models of audition usually involve static feedforward processing, whereas neural network models are usually adaptive. 
models of auditory cortical processing characterize transformations performed by cortical circuits, rather than detailed response properties of cortical neurons (see, e.g., K. Wang \& Shamma, 1995a). Most of these models involve nonadaptive processing of acoustic events. For example, Suga (1995) has modeled the auditory system of bats as a sequence of hierarchically organized filters. In contrast, neural network models of auditory cortical processing are generally adaptive (i.e., their response sensitivities are affected by training), making them useful for simulating experience-induced adaptation in the auditory cortex. These models either can be biologically based, focusing on the dynamics of cellular interactions (de Pinho \& Roque-da-Silva, 1999; Elliot \& Shadbolt, 1998; Palakal \& Wong, 1999; Sanchez-Montanes, Verschure, \& Konig, 2000), or may characterize cortical processing more abstractly (Armony et al., 1995; Myers, Gluck, \& Granger, 1995). Neural network models typically do not incorporate the types of transformations described by signal-processing models of audition. Inputs to neural networks usually consist of either binary patterns (Armony et al., 1995) or numerical values describing properties of sounds or neural responses (Bauer, Der, \& Herrmann, 1996; Guenther \& Gjaja, 1996; Palakal, Murthy, Chittajallu, \& Wong, 1995; Ritter, Martinez, \& Schulten, 1992).

Most computational models of auditory cortical processing in the past have involved nonadaptive filtering of inputs (Palakal et al., 1995; Suga, 1990; K. Wang \&
Shamma, 1995a) and thus have not accounted for the plasticity seen in adults. The few modelers that have attempted to characterize auditory plasticity have focused on changes in the sensitivities of individual neurons (Armony et al., 1995; Armony, Servan-Schreiber, Cohen, \& LeDoux, 1997; Armony, Servan-Schreiber, Romanski, Cohen, \& LeDoux, 1997; de Pinho, Mazza, \& Roque, 2000; Sanchez-Montanes et al., 2000). These models (with the exception of de Pinho, Mazza. \& Roque, 2000) do not address how the initial spatial organization of cortical response properties might affect cortical map reorganization.

Our approach was to take a neural network model that has served as the basis for many past cortical models (the self-organizing map) and integrate it with advanced signal processing models of auditory representation (Figure 2). The computational structure of the self-organizing map is straightforward and has been studied extensively (for reviews, see Kohonen, 1993, 1997; Ritter et al., 1992). This connectionist model has a highly flexible architecture that can be easily customized to model processing in sensory cortices (see, e.g., Erwin et al., 1995; Palakal et al., 1995; Ritter et al., 1992; Sirosh \& Miikkulainen, 1997; Swindale \& Bauer, 1998). Signalprocessing models of auditory representations have advanced considerably over the past decade (see, e.g., Meyer-Base \& Scheich, 1995; Pitton, Wang, \& Juang, 1996; Robert \& Eriksson, 1999; Tchorz \& Kollmeier, 1999; K. Wang \& Shamma, 1995a). Such models pro-

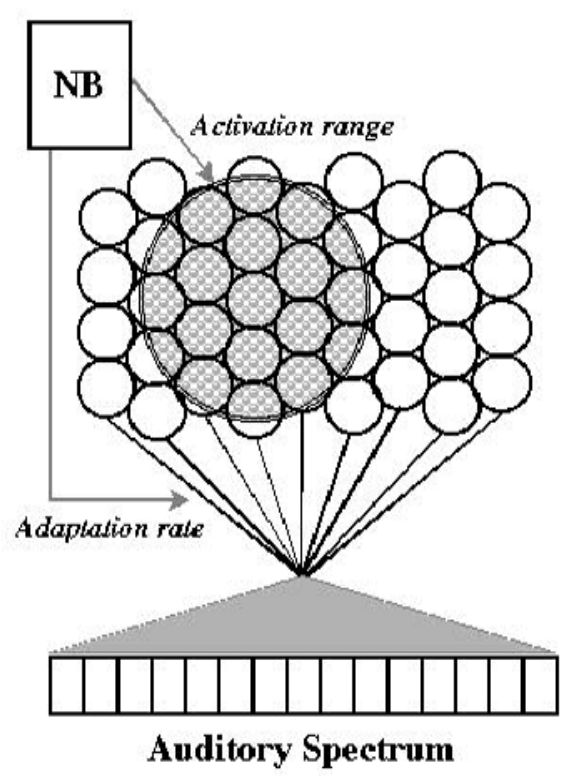

Self-organizing map model

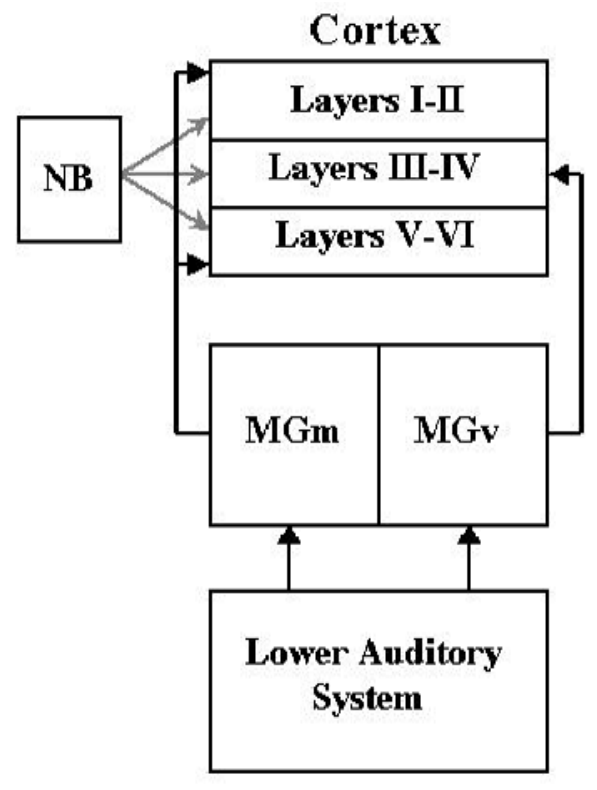

Weinberger et al. (1990) model

Figure 2. Models of basal forebrain modulation of cortical plasticity. In the self-organizing map model, modulation by neurons in the nucleus basalis $(\mathrm{NB})$ is simulated as changes in the area of the map activated by the most responsive node and/or by changes in the rate at which response properties adapt. In the Weinberger et al. (1990a, 1990b) model, NB modulation increases the excitability and adaptability of cortical neurons when behaviorally relevant events are occurring. 
vide a way to precisely characterize how auditory cortical response sensitivities are likely to change in response to learning (Mercado, Myers, \& Gluck, 2000).

To review, the self-organizing map consists of an $n$-dimensional matrix of nodes, each of which can potentially be activated by external inputs (represented as vectors). Inputs are sent to every node in the map via weighted connections (which are also represented as vectors). Whether a node is activated by a particular input depends on how well the input matches the weights associated with that node. The basic self-organizing maplearning algorithm is as follows (detailed mathematical formulations can be found in Kohonen, 1997). (1) Choose an input vector at random from the set of all input vectors; (2) measure the similarity between this input vector and all the weight vectors; (3) find the node with the most similar weight vector; (4) update the weights of this node and nearby nodes so that they are more similar to the input vector; and (5) continue this process until all the inputs have been presented to the map.

Input sets are typically presented to a self-organizing map several times. The number of surrounding nodes affected by activation of the best-matching node is defined in terms of a neighborhood function. Two parameters in the self-organizing map-learning algorithm can be used to control the adjustment of node weights: the learning rate function and the range of the neighborhood function (called the neighborhood size). Both parameters are usually decreased as training proceeds, so that early adjustments to weights are larger and have a more global effect than do later adjustments. The response properties of self-organizing maps become topographically organized with training, so that nearby nodes respond maximally to similar inputs.

Self-organizing maps with biologically based response characteristics can emulate the spatial organization of response properties observed in the auditory cortex, as well as the competitive adaptation currently theorized to underlie changes in auditory cortical organization.

\section{EFFECTS OF NEUROMODULATION ON AUDITORY CORTICAL REORGANIZATION}

Kohonen $(1987,1993)$ suggested that the adaptive processes underlying plasticity in biological neural networks may directly parallel the self-organizing map algorithm. He postulated that active cells can control adaptation in surrounding cells by extracellular transmission of diffuse chemical agents. The number of neighboring cells affected would depend on the extent and amount of chemical agent transmitted, providing a possible chemical implementation of the neighborhood function used in self-organizing maps.

Although diffuse intracortical transmission of neuromodulatory chemicals may provide a feasible mechanism for localized cortical plasticity (see, e.g., Ahissar et al., 1992; Cruikshank \& Weinberger, 1996b; Maldonado \& Gerstein, 1996; Zoli et al., 1998), neuromodula- tory systems originating outside the cortex provide an alternative source of diffuse chemical agents that can regulate reorganization in sensory cortices. Several neuromodulatory systems have been implicated in sensory processing and cortical plasticity (for reviews, see Edeline, 1999; Hasselmo, 1995; Zaborszky, Pang, Somogyi, Nadasdy, \& Kallo, 1999), three of which have been implicated in auditory cortical reorganization: the basal forebrain cholinergic system (Bakin \& Weinberger, 1996; Edeline, Hars, Maho, \& Hennevin, 1994; Hars, Maho, Edeline, \& Hennevin, 1993; Metherate \& Ashe, 1991, 1993), the mesencephalic dopaminergic system (Bao \& Merzenich, 2000; Stark \& Scheich, 1997), and the noradrenergic system (Edeline, 1995; Manunta \& Edeline, 1997). These neuromodulatory systems consist of concentrations of neurons with axons that project throughout the cortex. Many sites of neuromodulator release in the axons of these neurons are not associated with clearly identifiable synapses, suggesting that extrinsic neuromodulation may affect cortical activity, at least in part, through diffuse chemical control. Neuromodulators released from neurons in the basal forebrain and brain stem may thus mediate local, activity-dependent changes in cortical adaptability that are qualitatively similar to those controlled by neighborhood functions in self-organizing maps.

Past attempts at explaining the role that neuromodulators play in auditory cortical plasticity have focused primarily on modulation by neurons projecting from the nucleus basalis, a subpopulation of neurons in the basal forebrain. For example, Weinberger et al. (1990a; Weinberger et al., 1990b) proposed that acetylcholine released into the auditory cortex by the nucleus basalis amplifies inputs from the thalamus by enhancing postsynaptic activation throughout the cortex (see Figure 2). They suggested that synaptic connections from active thalamic cells to cortical pyramidal cells are strengthened and that nonactive synapses are weakened (i.e., adaptation occurs via Hebbian mechanisms). Dykes (1997) proposed a similar qualitative model to explain how basal forebrain modulation affects reorganization in the somatosensory cortex. In this model, acetylcholineinduced excitation combines with GABA-induced disinhibition to create a permissive cortical state that facilitates "LTP-like" synaptic plasticity.

These qualitative models are supported by recent neurophysiological studies showing that the nucleus basalis plays a prominent modulatory role in experiencedependent cortical plasticity (Bakin \& Weinberger, 1996; Baskerville, Schweitzler, \& Herron, 1997; Bjordahl, Dimyan, \& Weinberger, 1998; Dimyan \& Weinberger, 1999; Kilgard \& Merzenich, 1998a; Sachdev, Shao-Ming, Wiley, \& Edner, 1998). For example, when electrical stimulation of the nucleus basalis is repeatedly paired with presentation of a sound, auditory cortical neurons adapt so that the cortical area responsive to that sound is greatly expanded (Kilgard \& Merzenich, 1998a; Mercado, Shohamy, Orduña, Gluck, \& Merzenich, 2000). In contrast, basal forebrain lesions significantly reduce experience- 
dependent plasticity (Baskerville et al., 1997; Juliano, Ma, \& Eslin, 1991; Kilgard \& Merzenich, 1998a; Sachdev et al., 1998; Webster, Hanisch, Dykes, \& Biesold, 1991).

Researchers studying the role of basal forebrain modulation in cortical plasticity have typically focused on cholinergic effects. Increasing the amount of acetylcholine in the sensory cortex generally facilitates evoked responses (Ashe \& Weinberger, 1991; Edeline et al., 1994; Hars et al., 1993; Metherate \& Ashe, 1993; Metherate, Ashe, \& Weinberger, 1990; Metherate, Tremblay, \& Dykes, 1987; Tremblay, Warren, \& Dykes, 1990; Webster, Rasmusson, et al., 1991), whereas blocking or eliminating cholinergic effects reduces responsiveness (Delacour, Houcine, \& Costa, 1990; Edeline et al., 1994; Jacobs, Code, \& Juliano, 1991; Maalouf, Miasnikov, \& Dykes, 1998; Metherate \& Ashe, 1991, 1993). In addition, changes in cortical sensitivities can be induced by pairing the application of acetylcholine (or drugs that mimic cholinergic actions) with the presentation of tones (Ashe, McKenna, \& Weinberger, 1989; McKenna, Ashe, \& Weinberger, 1989; Metherate \& Weinberger, 1989, 1990; Shulz, Sosnik, Ego, Haidarliu, \& Ahissar, 2000). Cholinergic effects on cortical plasticity are activity dependent. For example, basal forebrain stimulation without the presentation of sound does not lead to long-lasting facilitation of evoked responses (Bakin \& Weinberger, 1996; Edeline et al., 1994; Hars et al., 1993).

Although cholinergic neurons in the nucleus basalis are a primary source of cortical acetylcholine, recent anatomical studies suggest that cholinergic neurons represent a relatively small fraction of the modulatory cells projecting from the nucleus basalis to sensory cortices. The majority of projecting neurons appear to be either GABAergic or peptidergic (Gritti, Mainville, Mancia, \& Jones, 1997; Zaborszky et al., 1999). Auditory cortical sensitivities can be substantially modified by blocking the effects of GABA (Foeller, Vater, \& Koss1, 2000; Schulze \& Langner, 1999; J. Wang, Caspary, \& Salvi, 2000), providing support for Dykes's (1997) proposal that GABAergic modulation can significantly affect cortical reorganization (see also Giorgetti et al., 2000; Jiminez-Capdeville, Dykes, \& Myasnikov, 1997). The effects of peptides on auditory cortical plasticity, if any, are unknown.

In summary, neuromodulators released in the auditory cortex by basal forebrain and brain stem neurons appear to control experience-dependent changes in neuronal sensitivities. Although the mechanisms underlying neuromodulatory effects on cortical plasticity remain conjectural, electrophysiological measurements provide clear evidence that basal forebrain neurons, in particular, modulate both the activity and the adaptability of auditory cortical neurons. Because the roles of other neuromodulatory systems in cortical plasticity have been investigated in much less detail, the present simulations focus on modeling the effects of basal forebrain modulation on experiencedependent changes in cortical representations.

\section{MODELING BASAL FOREBRAIN MODULATION}

How can the effects of basal forebrain modulation be incorporated within our model of auditory cortical processing? Assume that, in certain contexts, basal forebrain neurons control when, where, and to what extent cortical neurons change their response properties. In the selforganizing map, where is determined by input features and the neighborhood size, when is determined by input features and the learning rate, and to what extent is determined by the neighborhood size and the learning rate. Thus, adjusting the neighborhood size and the learning rate in our simulated auditory cortex affects reorganization in ways that qualitatively parallel the activity-dependent effects of basal forebrain modulation on reorganization in the auditory cortex.

In the qualitative models proposed by Weinberger et al. (1990a, 1990b) and Dykes (1997) (see also Gao \& Suga, 2000), neuromodulators affect auditory cortical plasticity by changing the likelihood that signals from cochlear receptors and/or intracortical connections will activate cortical neurons. Increased or decreased activation within particular cortical regions is then hypothesized to control (via Hebbian processes) how synaptic connections in those regions are modified (see also Ahissar, Abeles, Ahissar, Haidarliu, \& Vaadia, 1998; Fregnac \& Shulz, 1999). Our model dissociates this sequence of events into two subprocesses: excitation and adaptation. When neuronal excitability is increased in the auditory cortex, larger populations of neurons respond to the presentation of a pure tone (see, e.g., J. Wang et al., 2000). This increase in responsive area is similar to that produced by increasing the intensity of a tone (Bakin, Kwon, Masino, Weinberger, \& Frostig, 1996). Paralleling these effects, we model changes in excitability in terms of changes in neighborhood size. Increases in neuronal adaptability have been qualitatively modeled as changes in synaptic plasticity based on modified Hebbian rules. Similarly, we use changes in learning rate to model changes in synaptic plasticity induced by basal forebrain modulation.

\section{Method}

In the present study, we simulated plasticity in primary auditory cortex, using the SOM Toolbox, developed by students of Kohonen at the Laboratory of Information and Computer Science in the Helsinki University of Technology. The SOM Toolbox is a software library for Matlab 5 that implements the self-organizing map algorithm. We used an $11 \times 20$ node two-dimensional map to grossly approximate the spatial organization of the primary auditory cortex in various mammals (as described in Aitkin, 1990; Recanzone, Schreiner, Sutter, Beitel, \& Merzenich, 1999; Schreiner, 1998; K. Wang \& Shamma, 1995a) and for computational tractability. The local topography of the map was hexagonal-that is, each node was surrounded by 6 other nodes. A Gaussian-shaped neighborhood function was used. Individual nodes in the self- 
organizing map simulate cortical regions, rather than individual neurons. Recent research suggests that individual neurons within local regions respond similarly to acoustic stimuli and that it is likely that these clusters of neurons serve as coherent functional processing units (see, e.g., deCharms \& Merzenich, 1996; M. E. Diamond \& Ebner, 1990; X. Wang, Merzenich, Beitel, \& Schreiner, 1995).

The weights associated with each node in a selforganizing map are typically initialized either to random values or to values chosen on the basis of the linear subspace spanned by the eigenvectors of the input data set. This is not the normal initial state of the primary auditory cortex in adult mammals, however. Sensitivities in the mammalian auditory cortex are moderately predictable. Researchers have mapped out how cortical neurons in particular areas respond to different spectral and temporal components of sounds (for reviews, see Ehret, 1997; Merzenich \& Schreiner, 1992; Schreiner, Read, \&
Sutter, 2000; K. Wang \& Shamma, 1995b). We initialized the self-organizing map to emulate previously reported spatial properties and spectral sensitivities of the primary auditory cortex (see Figure 3). Specifically, response sensitivities were initialized to be cochleotopic (i.e., the topography of cortical sensitivities parallels the spatial arrangement of cochlear receptors), and nodes in the inner regions of the map were initialized to respond to a narrower band of frequencies than do nodes in the outer edges (see, e.g., Recanzone et al., 1999; Schreiner, 1998; Schreiner et al., 2000; K. Wang \& Shamma, 1995a). Note that the organization of map response properties in our model is grossly oversimplified in comparison with actual cortical sensitivities, which can vary greatly across individuals, species, and recording contexts.

Simulations were performed to examine how map reorganization was affected by variation in four parameters: number of input exposures, learning rate, neighborhood size, and number of different tones presented to a map
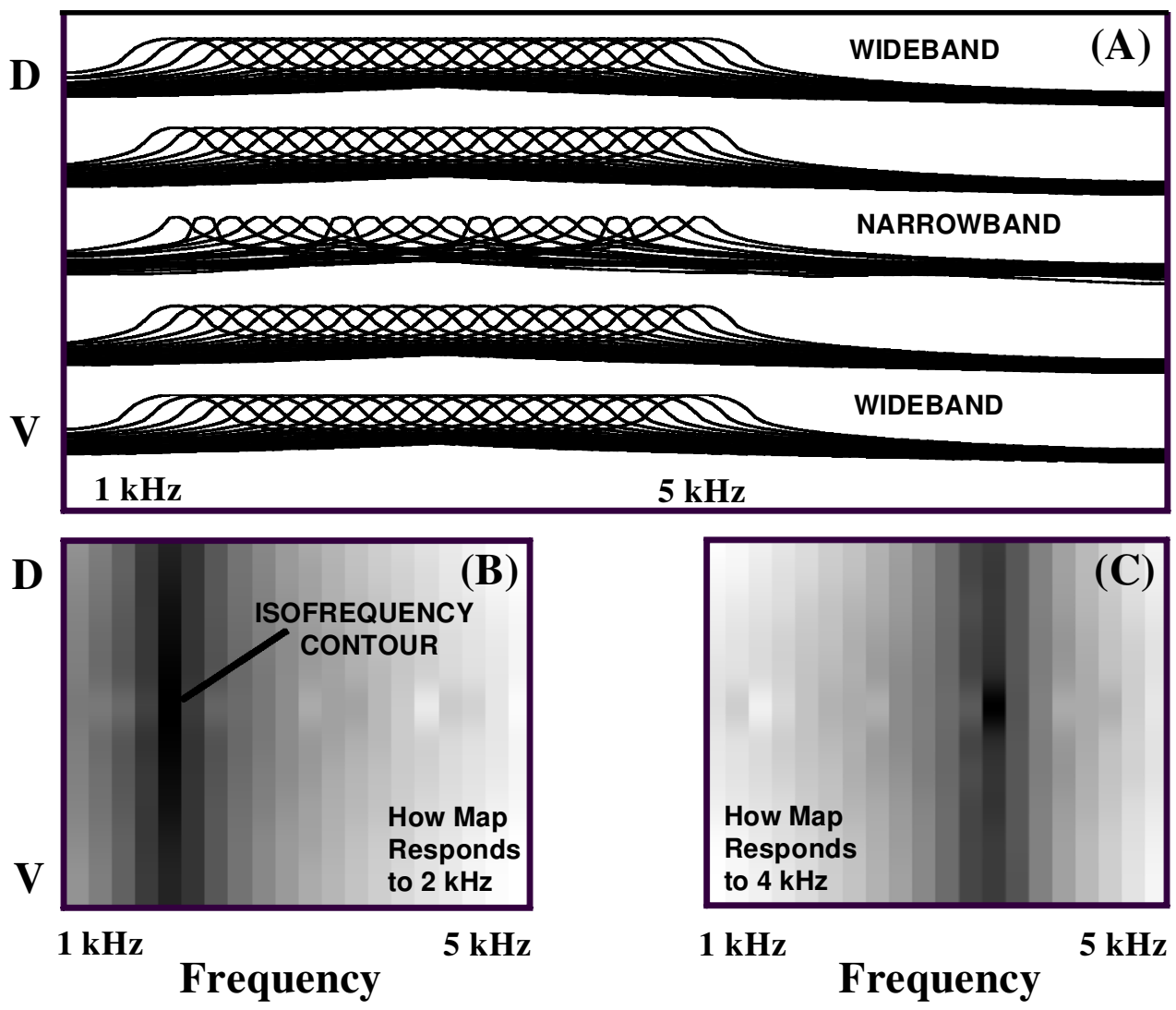

Figure 3. Frequency response properties of initialized map nodes. (A) Each curve represents the frequency response of an initialized node in the self-organizing map ( 5 of the 11 rows of nodes are shown). Middle rows respond to a narrower band of frequencies than do nodes located either dorsal (D) or ventral (V) to the middle. This organization makes the initial response properties of the map symmetrical, reflecting the "redundant" spectral sensitivities measured electrophysiologically in the auditory cortex. (B) Activity levels across the map in response to a $2-\mathrm{kHz}$ tone (darker regions correspond to higher activity levels). The dark vertical band indicates a column of nodes activated by the 2 -kHz tone; similar bands (called isofrequency contours) have been observed in the auditory cortex. (C) Activity levels in response to a $4-\mathrm{kHz}$ tone. 
Table 1

Parameter Values Used in Simulations of Auditory Cortical Reorganization

\begin{tabular}{lcccc}
\hline Level & Exposures & Learning Rate & Neighborhood Size & Tones \\
\hline Low & 1,250 & 0.005 & 2 & 1 \\
Medium & 2,500 & 0.05 & 5 & 2 \\
High & 5,000 & 0.5 & 10 & - \\
\hline
\end{tabular}

(see Table 1). Increases in the number of times a map was exposed to a particular input simulate increases in the number of times a particular sound is paired with activation of basal forebrain neurons. The learning rate parameter controls the extent to which connections to active nodes are changed in response to each input presentation. Increases in this parameter simulate enhanced neural adaptability (see also Myers et al., 1996; Myers et al., 1998). Neighborhood size determines the number of surrounding nodes activated by a "winning" node. Increases in this parameter simulate enhanced neural excitability (see also Piepenbrock \& Obermayer, 2000).

Previous simulations of experience-dependent auditory cortical plasticity have typically focused on changes in the response properties of single cells induced by classical conditioning (Armony et al., 1995; de Pinho, Mazza, $\&$ Roque, 2000). Such changes can be produced after a small number (5-30) of training trials (Bakin \& Weinberger, 1990; Edeline \& Weinberger, 1993a). Although observations of rapid, learning-induced changes in cortical sensitivities suggest that concomitant rapid changes in the topography of auditory cortical maps might also be occurring (Weinberger et al., 1990a, 1990b), this possibility has yet to be verified experimentally. Changes in the topographical organization of representational maps have been demonstrated, however, after larger numbers (600-40,000+) of conditioning trials (Gonzalez-Lima \& Scheich, 1986; Recanzone, Schreiner, \& Merzenich, 1993 ) and after pairing basal forebrain stimulation with the presentation of tones for thousands of trials (Kilgard $\&$ Merzenich, 1998a). Our simulations focus on reorganization in simulated auditory cortical maps that are induced by relatively large numbers of repeated exposures to a particular sound. It is important to note, however, that the number of training iterations used in our simulations currently cannot be directly equated either with the number of conditioning trials in a behavioral task or with the number of pairings used in a stimulation experiment. Thus, 1,000 training iterations could potentially produce changes comparable with those observed after only 10 conditioning trials.

To assess the utility of our model, we compared changes observed in our simulations with changes in cortical sensitivities induced by pairing presentations of tones with basal forebrain stimulation in rats. Specifically, parameters were modulated in an attempt to grossly replicate changes in the organization of the auditory cortex reported by Kilgard and Merzenich (1998a). Kilgard and Merzenich (1998a) stimulated rats 300-500 times a day, for
7-25 days, for a total of about 2,500-12,500 trials. For some rats, stimulation was paired with the presentation of a $9-\mathrm{kHz}$ tone in all the trials, whereas for other rats, stimulation was paired with a $9-\mathrm{kHz}$ tone in half the trials and a $19-\mathrm{kHz}$ tone in the remaining trials (trials of each type were randomly intermixed). Similarly, self-organizing maps (with varying learning rates and neighborhood sizes) were exposed to either a single $2-\mathrm{kHz}$ pure tone or two different tones ( $2 \mathrm{kHz}$ and $4 \mathrm{kHz}$ ), 1,250-5,000 times.

Changes in the sensitivities of self-organizing maps were measured in terms of differences in the response characteristics of trained nodes relative to initialized nodes. These measurements allow qualitative comparisons to be made between the results of our simulations and the data reported by Kilgard \& Merzenich (1998a) and quantitative comparisons to be made across simulations. Our simulations also allow us to make detailed predictions about how the topography of neuronal sensitivities should change, depending on how basal forebrain modulation affects neural excitability and adaptability.

\section{AUDITORY CORTICAL PLASTICITY: EMPIRICAL AND MODEL DATA}

\section{Changes in Spectral Receptive Fields in Rats and Neural Networks}

Plasticity in the auditory cortex is commonly described in terms of changes in neural firing rates induced by presentation of short-duration tonal sounds (tone pips). Neurons typically fire most strongly to tones within a particular range of frequencies. Thus, each neuron can be described in terms of its frequency response function (or spectral receptive field). The frequency of the lowest intensity tone pip that consistently produces a change in firing rate is called the characteristic frequency, and the frequency of the tone pip that produces the greatest change in firing rate is called the best frequency.

Several learning- and stimulation-induced changes in spectral receptive fields have been described. These changes are often reported relative to a particular sound (e.g., a tone pip of a particular frequency) that has acquired relevance or irrelevance as a predictor of an aversive event (e.g., an electric shock) or basal forebrain stimulation. For example, most studies of learning-induced plasticity have compared changes in neuronal responses to a conditioned stimulus (e.g., a tone pip that has been repeatedly paired with shock) with changes in responses to other frequencies that have no predictive relevance (Bakin \& Weinberger, 1990; Edeline, NeuenschwanderEl Massioui, \& Dutrieux, 1990; Edeline \& Weinberger, 1993b; Ohl \& Scheich, 1996, 1997; Weinberger, Javid, \& Lepan, 1993). A smaller number of studies have examined changes in responses induced by pairing several different sounds with basal forebrain activation (Kilgard \& Merzenich, 1998a, 1998b).

Details of previous findings on experience-induced changes in receptive fields will not be described here, because these data have been extensively reviewed (see, 
e.g., Ahissar \& Ahissar, 1994; Edeline, 1999; Kaas, 1996; Rauschecker, 1999; Weinberger, 1993, 1995, 1997, 1998a; Weinberger \& Bakin, 1998; Weinberger \& Diamond, 1987). A wide variety of experience-dependent changes in spectral sensitivities can be induced by either basal forebrain stimulation or behavioral training, including (1) increased responses to a conditioned tone pip (Bakin \& Weinberger, 1990; D. M. Diamond \& Weinberger, 1986; Edeline \& Weinberger, 1993a; Weinberger et al., 1993); (2) decreased responses to a conditioned tone pip (D. M. Diamond \& Weinberger, 1984; Kraus \& Disterhoft, 1982; Ohl \& Scheich, 1996, 1997; Weinberger, Hopkins, $\&$ Diamond, 1984); (3) increased responses to frequencies surrounding a conditioned or stimulated tone pip (Bjordahl et al., 1998; D. M. Diamond \& Weinberger, 1989; Metherate \& Weinberger, 1989; Ohl \& Scheich, 1996, 1997); (4) decreased responses to frequencies other than that of a conditioned tone pip (Bakin, South, \& Weinberger, 1996; Bakin \& Weinberger, 1990; Edeline \& Weinberger, 1993b; Weinberger et al., 1993); (5) changes in the size (e.g., bandwidth) of the receptive field (Edeline \& Weinberger, 1993b; Kilgard \& Merzenich, 1998a; Weinberger et al., 1993); and (6) general increases/decreases in neural responsiveness (Bakin, Lepan, \& Weinberger, 1992; Bakin \& Weinberger, 1990;
D. M. Diamond \& Weinberger, 1989; Edeline \& Weinberger, 1993a). Cases in which neurons show no statistically significant changes in responsiveness to a conditioned tone pip have also been observed (see, e.g., Edeline \& Weinberger, 1993b; Weinberger et al., 1984). Although the wide range of reported changes in receptive fields can be attributed, in part, to differing methodologies, many varieties of changes have been reported in individual studies. Experience-induced changes in neuronal response properties can also fluctuate over time (Bjordahl et al., 1998; Ohl \& Scheich, 1996).

Not surprisingly, changes in the spectral response properties of nodes in simulated auditory cortices were much less variable than those observed experimentally. The most common changes observed included increased sensitivity to a targeted frequency and decreased sensitivity to tone pips that were not presented to the map. Figure 4A shows how individual nodes in a simulated cortex respond after being exposed to a $2-\mathrm{kHz}$ pure tone for 2,500 trials, using a high learning rate and a low neighborhood size (i.e., simulating enhanced neural adaptability without enhanced excitability). In this scenario, training changed the response properties of $51 \%$ of the nodes. For comparison, Figure 5A shows how the map responded after being exposed to a $2-\mathrm{kHz}$ pure tone for
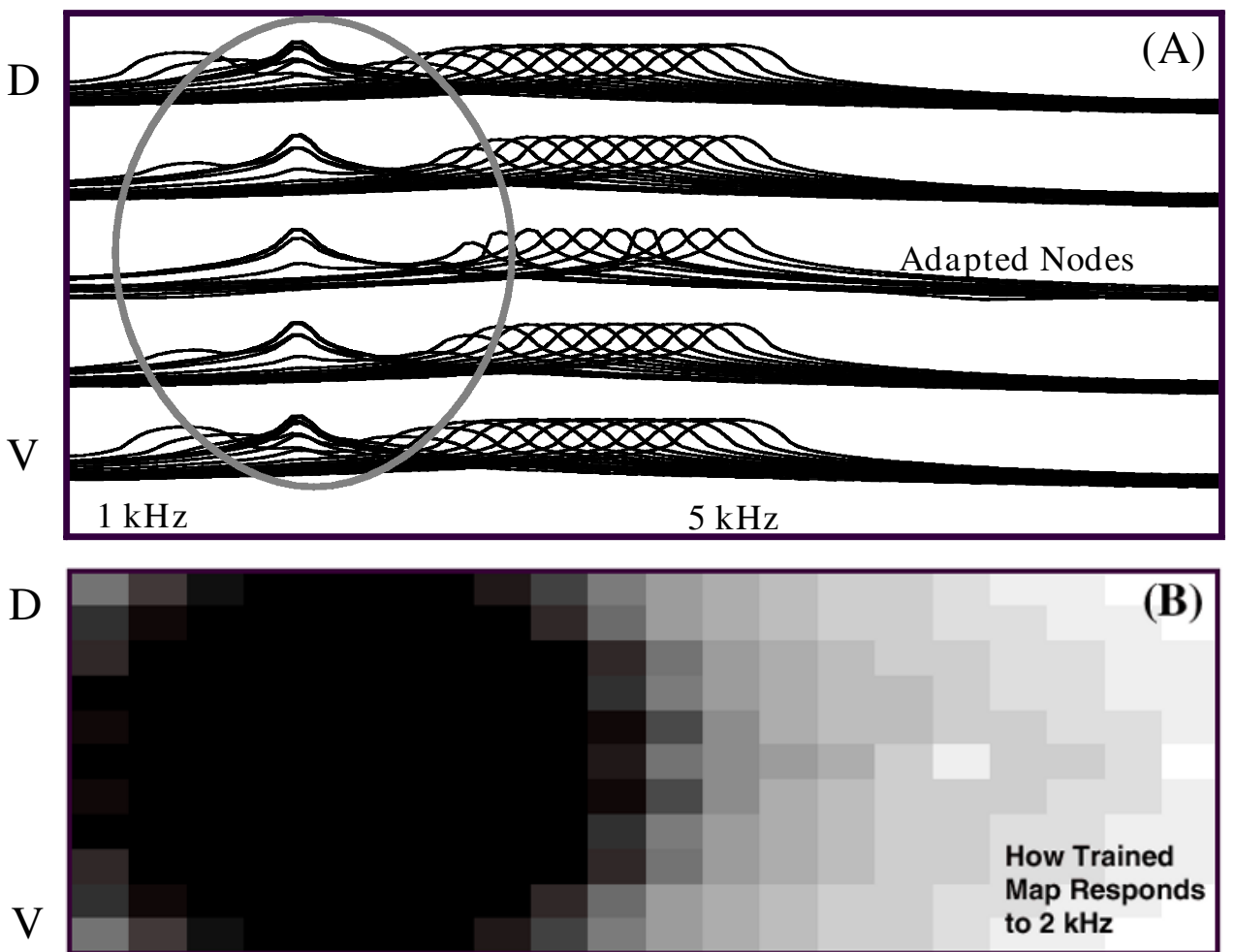

$1 \mathrm{kHz}$

Frequency

$5 \mathrm{kHz}$

Figure 4. (A) Response properties for a map trained with a $2-\mathrm{kHz}$ pure tone for 2,500 trials, using a low neighborhood size and a high learning rate. Note that the responses of nodes on the right side of the map have not been affected by training. (B) A substantially increased number of nodes (10 times more than in the initial map) now respond strongly to $2 \mathrm{kHz}$. 


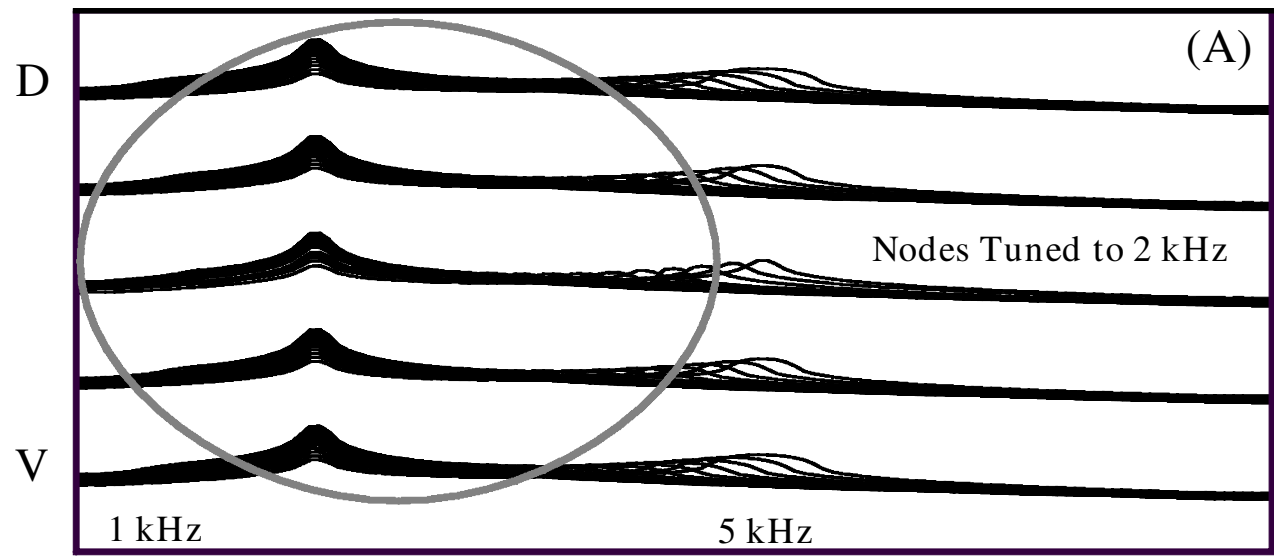

$\mathrm{D}$

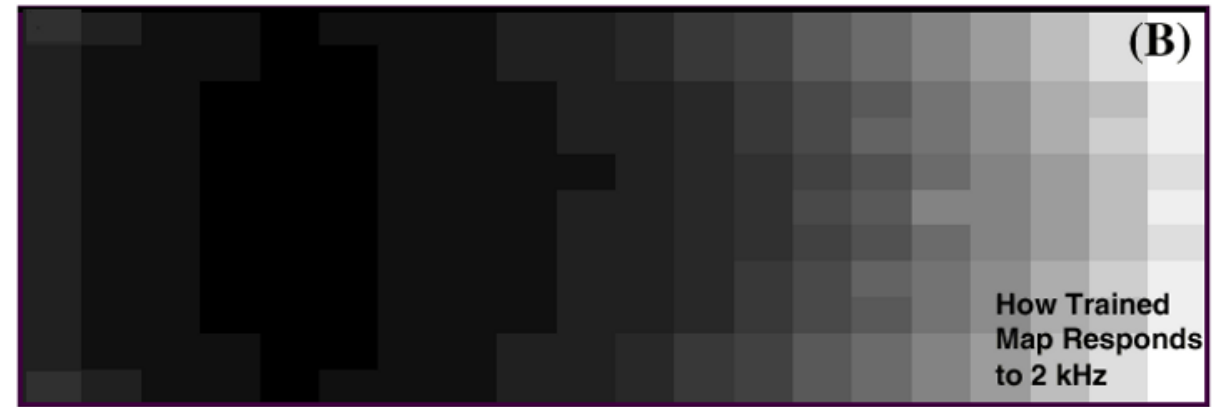

$1 \mathrm{kHz}$

Frequency

$5 \mathrm{kHz}$

Figure 5. (A) Response properties for a map trained with a $2-\mathrm{kHz}$ pure tone for 1,250 trials, using a high neighborhood size and a medium learning rate. (B) Over $80 \%$ of map nodes now respond best to $2 \mathrm{kHz}$.

1,250 trials when there was a lower learning rate and a high neighborhood size (i.e., simulating enhanced neural excitability with moderately enhanced adaptability). Even though this map was exposed to only half as many inputs as the map shown in Figure 4A, most $(82 \%)$ of the nodes responded strongly to $2 \mathrm{kHz}$. Overall, increases in excitability led to quicker and more extensive changes in response properties than did increases in learning rate (Figure 6).

In all of our simulations, we observed that the frequency response characteristics of individual nodes flattened (e.g., threshold and bandwidth increased) as they became "retuned." Consequently, responses to characteristic frequencies decreased, whereas responses to the frequency paired with stimulation increased (Figure 7A). When neighborhood size was high, individual nodes showed a gradual decrease in flattening of the receptive field as a function of distance from the target frequency (Figure 5A). In contrast, a low neighborhood size led to more discrete changes in the flatness of receptive fields (see Figure 4A). Overall, such changes in spectral response properties are consistent with those observed in other neural network models of learning-induced auditory cortical plasticity (e.g., Armony et al., 1995).

Figures 8, 9, and 10 illustrate how maps respond after being exposed to both a $2-\mathrm{kHz}$ (625 trials) and a $4-\mathrm{kHz}$ (625 trials) pure tone (exposures to each of the two tones were randomly intermixed). When there was a low neighborhood size, nodes at the dorsal and ventral edges of the map reorganized more slowly than inner nodes located an equivalent or greater distance from "winning" nodes (i.e., nodes with characteristic frequencies matching the stimulated frequencies; see Figures 8A and 10A). In contrast, simulations in which there was a high neighborhood size showed no correlation between node position and speed of adaptation for an equal number of exposure trials (Figure 9A). All of the simulations with two tones consistently showed a tendency toward bimodally distributed frequency response characteristics. When neighborhood size was low, some nodes became tuned to $2 \mathrm{kHz}$, others became tuned to $4 \mathrm{kHz}$, and a few nodes developed multipeaked responses (see Figure 8A). When neighborhood size was high, all the nodes became tuned to both 2 and $4 \mathrm{kHz}$ (see Figure 9A). Simulations consistently showed reduced responsiveness to frequencies between 2 and $4 \mathrm{kHz}$, especially at $3 \mathrm{kHz}$, the linear midpoint between these two frequencies.

\section{Map Reorganization in Rats and Neural Networks}

Relatively few studies have examined global changes in the spatial distribution of response properties across the auditory cortex induced by learning or basal forebrain stimulation. This is somewhat surprising, given the large amount of research devoted to describing the spatial or- 


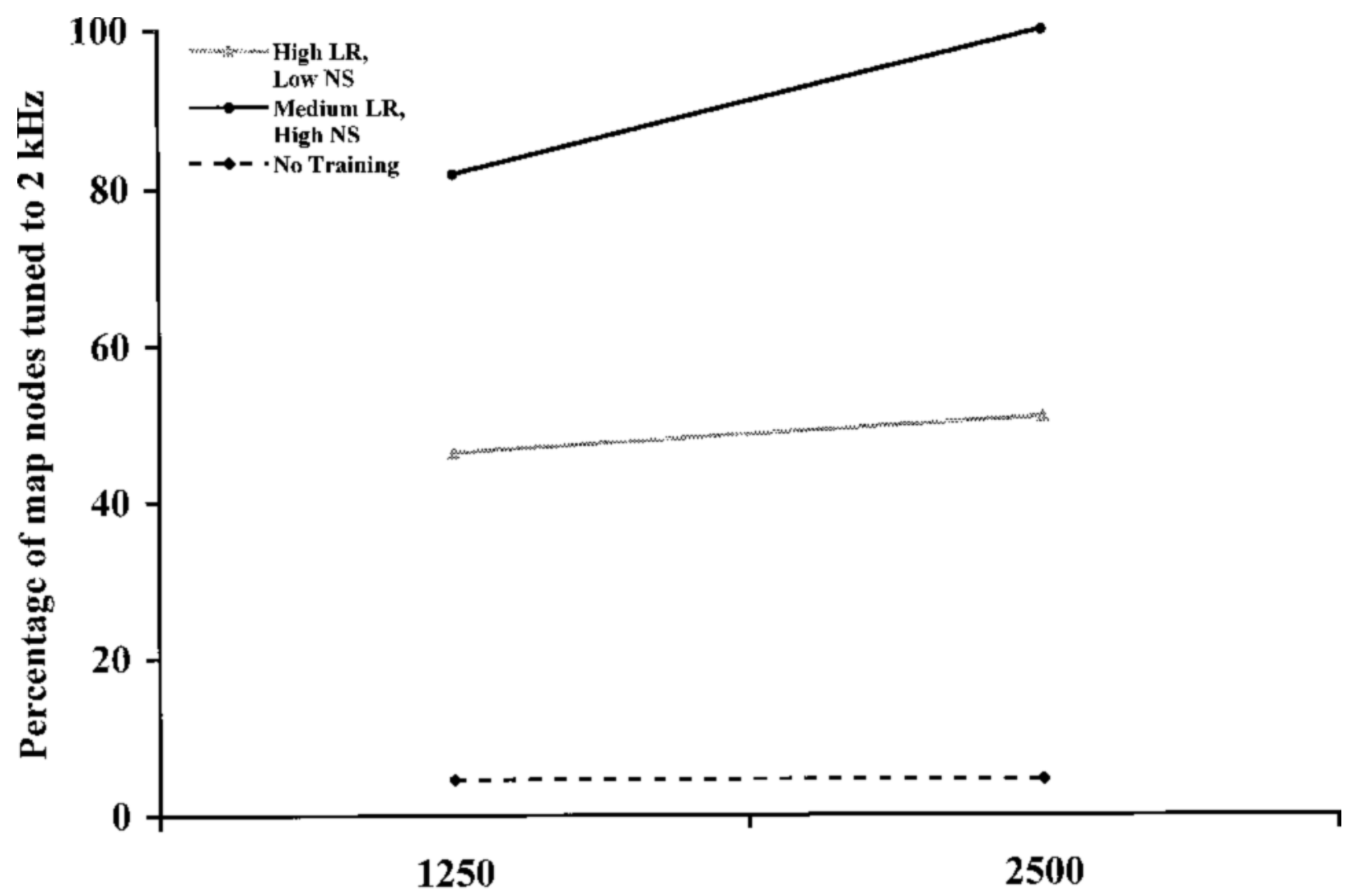

Number of exposures

Figure 6. Increases in excitability (neighborhood size) lead to larger increases in the number of nodes responding best to the target frequency. LR, learning rate; NS, neighborhood size.

ganization of auditory cortical sensitivities (for reviews, see Aitkin, 1990; Ehret, 1997; Schreiner et al., 2000).

Maps of cortical sensitivities have typically been measured either anatomically or electrophysiologically. Response sensitivities in the auditory cortex can be mapped anatomically, using a variety of labeling techniques (e.g., $\mathrm{C}$-fos, 2-DG). Labeling can reveal which neurons in the cortex have been activated in response to the presentation and conditioning of particular acoustic stimuli. Changes in cortical maps can be assessed by comparing patterns of activity in naive subjects with those of trained subjects (for reviews, see Edeline, 1999; Scheich, Stark, Zuschratter, Ohl, \& Simonis, 1997). Changes in cortical maps can also be measured by recording firing patterns from electrodes inserted throughout the auditory cortex (Kilgard \& Merzenich, 1998a; Recanzone et al., 1993) or using optical imaging techniques (Bakin, Kwan, et al., 1996). Recently, noninvasive techniques, such as magnetoencephalography (MEG), have been used to measure changes in auditory cortical sensitivities in humans (Cansino \& Williamson, 1997; Molchan, Sunderland, McIntosh, Herscovitch, \& Schreurs, 1994; Morris, Fris- ton, \& Dolan, 1998; Pantev \& Lutkenhoner, 2000; Pantev, Wollbrink, Roberts, Engelien, \& Lutkenhoner, 1999).

Studies of map reorganization based on anatomical techniques have shown that aversively conditioning gerbils with a $1-\mathrm{kHz}$ tone (e.g., by pairing the tone with a shock) leads to increased cortical sensitivity to frequencies below 1 kHz (Gonzalez-Lima \& Scheich, 1986; Scheich et al., 1997); such shifts were not observed after avoidance conditioning, in which gerbils could escape the aversive stimulus. However, both avoidance and aversion conditioning led to increased cortical responsiveness, relative to gerbils that were habituated to a $1-\mathrm{kHz}$ tone. Electrophysiological studies with monkeys showed that training on a frequency discrimination task led to increases in the cortical area responsive to behaviorally relevant frequencies (Recanzone et al., 1993). Basal forebrain stimulation paired with tone presentations in rats led to massive reorganization of cortical topography so that the majority of neurons responded to the stimulated tone (Kilgard \& Merzenich, 1998a). Changes in cortical responsiveness have also been observed, using MEG 


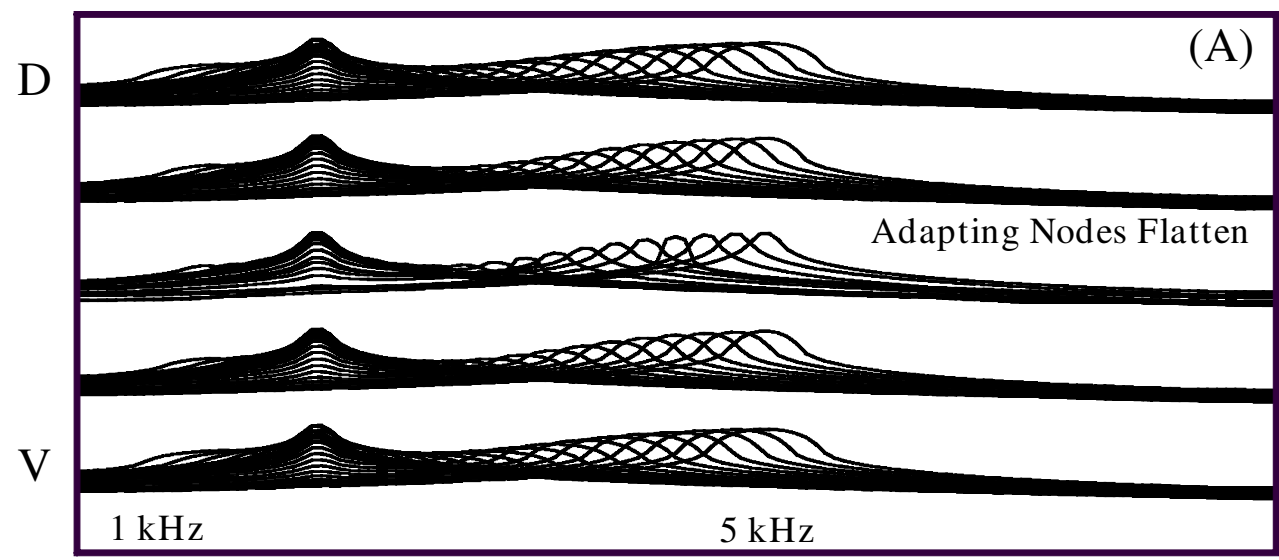

$\mathrm{D}$

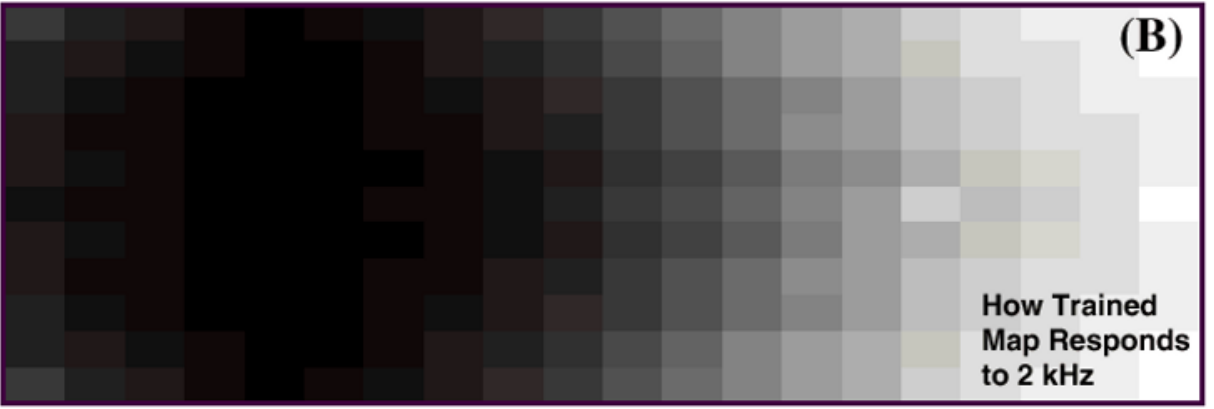

$1 \mathrm{kHz}$

Frequency

$5 \mathrm{kHz}$

Figure 7. (A) Response properties for a map trained with a $2-\mathrm{kHz}$ pure tone for 1,250 trials, using a medium neighborhood size and a medium learning rate. Note how responses to characteristic frequencies decrease, whereas responses to the target frequency increase. (B) Comparison with Figures $5 \mathrm{~A}$ and $6 \mathrm{~A}$ shows that intermediate parameter values give rise to intermediate levels of reorganization.

during auditory discrimination tasks (Cansino \& Williamson, 1997) and after spectral deprivation (Pantev et al., 1999). Such changes have not, however, been directly related to changes in the organization of auditory cortical maps.

Reorganization in self-organizing maps after training with one tone was generally comparable with that seen in electrophysiological experiments. The map area responsive to the target tone increased with training; the extent to which responsive regions expanded and the number of exposures necessary to reach a particular level of expansion were primarily determined by the neighborhood size. Increasing the neighborhood size greatly reduced the number of exposures needed for maps to massively reorganize across a wide range of learning rates (Figure 6). Figure 4B shows how the map responds after being exposed to a $2-\mathrm{kHz}$ pure tone for 2,500 trials when there was a high learning rate and a low neighborhood size. Note that (1) the area of the map responding to $2 \mathrm{kHz}$ has expanded considerably, (2) regions of the map responding to lower frequencies have compressed, and (3) the distribution of nodes sensitive to $2 \mathrm{kHz}$ is roughly circular. Much less reorganization occurs when neither learning rate nor neighborhood size is high (Figure 10B). In com- parison, Figure 5B shows how the map responded after being exposed to a $2-\mathrm{kHz}$ pure tone for 1,250 trials when there was a lower learning rate and a high neighborhood size. In this map, most of the nodes responded best to $2 \mathrm{kHz}$, and the nodes most sensitive to higher frequencies appear to have shifted outward.

Simulations involving the presentation of two tones produced reorganization that was inconsistent with empirical observations. For example, in a map that was exposed to both a $2-\mathrm{kHz}$ (625 trials) and a $4-\mathrm{kHz}$ (625 trials) pure tone, with a high learning rate and a low neighborhood size (Figure 8B), reorganization was focused at target frequencies, and a narrow region between 2 and $4 \mathrm{kHz}$ was not tuned to either tone. Similarly, a map exposed to the same inputs, when a high learning rate and a high neighborhood size were used (Figure 9B), showed a predominance of nodes most sensitive to 2 and $4 \mathrm{kHz}$ and a large region between 2 and $4 \mathrm{kHz}$ not tuned to either tone. In this map, unlike the map shown in Figure 8B, the nodes most sensitive to 2 and $4 \mathrm{kHz}$ migrated to opposite corners. In contrast, when two tones are paired with basal forebrain stimulation in rats, cortical maps reorganize in such a way that most neurons respond best either to one of the stimulated frequencies or, surprisingly, to 

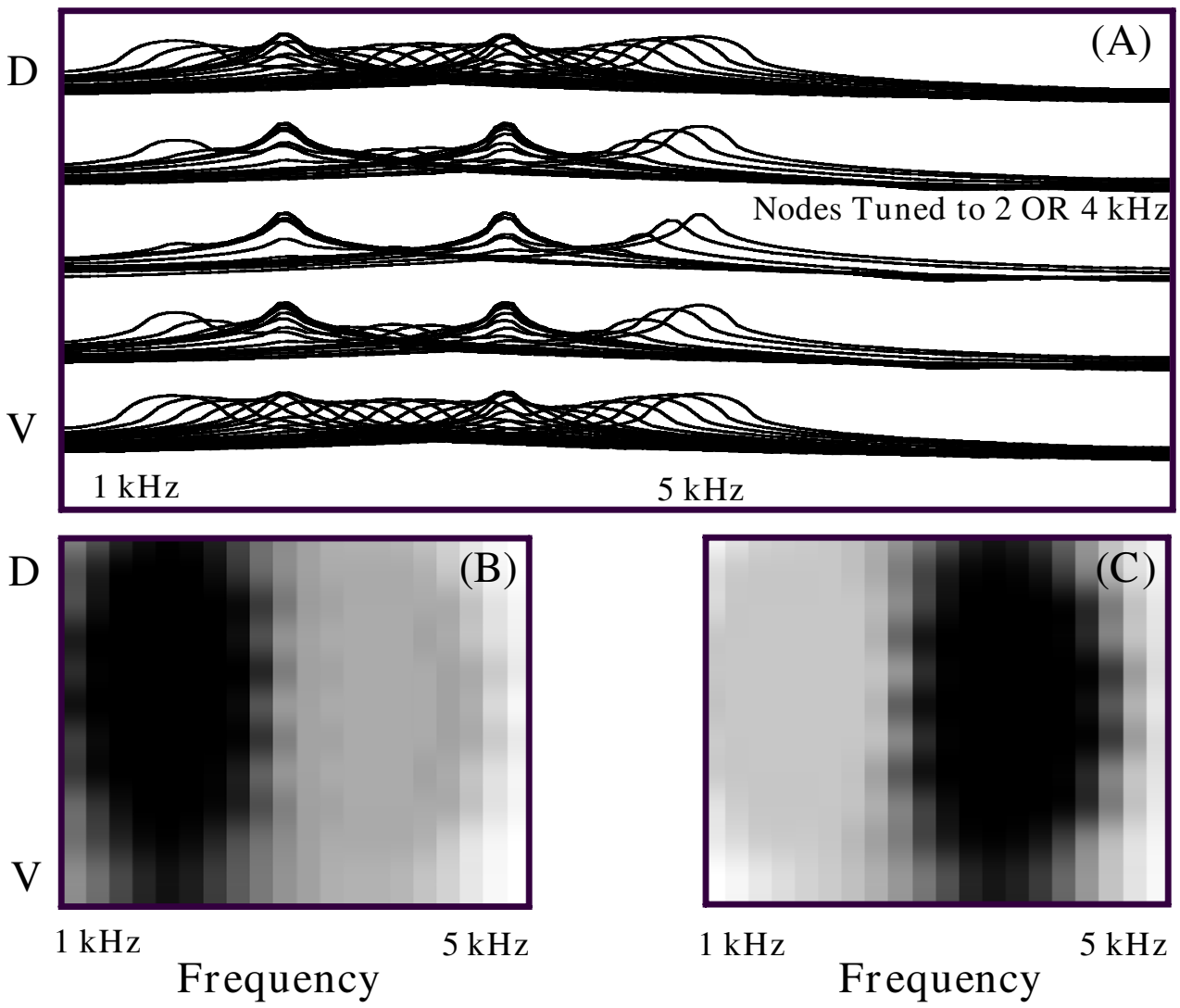

Figure 8. (A) Response properties for a map trained with a $2-\mathrm{kHz}$ tone (625 trials) and a 4-kHz tone (625 trials), using a low neighborhood size and a high learning rate. (B) and $(C)$ show that changes in sensitivity are focused around the original nodes responding best to $2 \mathrm{kHz}$ and $4 \mathrm{kHz}$, respectively.

frequencies between the two stimulated frequencies (Kilgard \& Merzenich, 1998a).

\section{DISCUSSION}

In this paper, we directly tested Kohonen's (1993) suggestion that the effects of neuromodulatory chemicals on cortical processing could be modeled as variations in the neighborhood functions of self-organizing maps. Specifically, we attempted to develop a self-organizing map model of the auditory cortex that could be reorganized in ways comparable with the cortical reorganization induced by pairing the presentation of sound with basal forebrain activation.

The results of our simulations demonstrate that a selforganizing map can be used to quantitatively characterize the effects of enhanced synaptic adaptability and neural excitability on auditory cortical reorganization. We found that node excitability (as controlled by neighborhood size) was the primary determinant of the number of exposures required before massive reorganization was evident in maps. When excitability was high, more nodes were adapted during each trial. As nodes became sensitized to the target frequency, the likelihood that a new best-responding node would emerge increased. Even when adaptation was rela- tively low, numerous nodes were affected by training. Varying either the adaptability or the excitability of nodes led to predictable changes in the response features of reorganized maps (summarized in Table 2).

Simulations of auditory cortical reorganization induced by training or stimulation with a single tone produced results generally comparable with those observed experimentally (e.g., by Recanzone et al., 1993) and in prior computational studies. Map nodes became tuned to the target frequency, and the area of map representing that frequency expanded considerably. Interestingly, changes in response properties occurred first in the regions of the map where the most sharply tuned nodes were located (see, e.g., Figure 10). We are not aware of any reports of correlations between the initial bandwidth of a receptive field and the probability (or rate) of retuning. We were also unable to find any reports describing differential potential for rapid plasticity as a function of spatial position within the auditory cortex. Experiments examining whether such correlations do or do not exist could provide important evidence for testing current models of auditory cortical plasticity.

Simulations of changes in response sensitivities induced by pairing stimulation with two tones revealed a limitation in our model. In empirical tests, pairing basal 

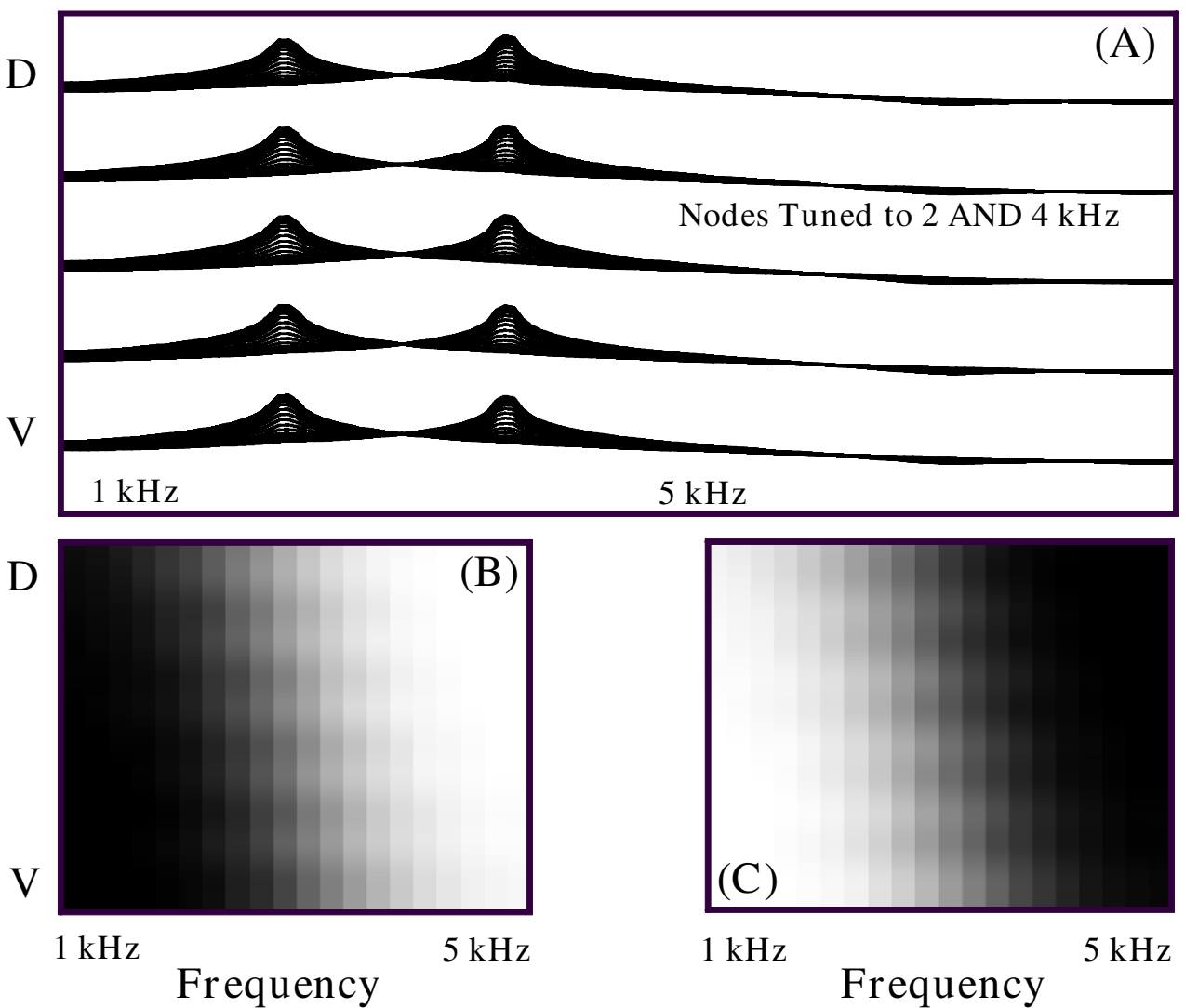

Figure 9. (A) Response properties for a map trained with a $2-\mathrm{kHz}$ tone (625 trials) and a 4-kHz tone (625 trials), using a high neighborhood size and a high learning rate. Sensitivities are clearly bimodal. (B) and (C) show that map nodes most sensitive to $2 \mathrm{kHz}(\mathrm{B})$ and $4 \mathrm{kHz}(\mathrm{C})$ have migrated to opposite corners (i.e., the symmetry of response properties has shifted).

forebrain stimulation with two tones led to unimodally distributed neural response characteristics (Kilgard \& Merzenich, 1998a), whereas in the present simulations, this did not occur. In addition, pairing basal forebrain stimulation with two tones sharpens tuning in the auditory cortex (Kilgard \& Merzenich, 1998a); we did not observe this effect in our simulations with two tones. These disparities suggest that stimulation-induced plasticity in the auditory cortex is constrained in ways not accounted for by the competitive Hebbian learning algorithm used in self-organizing maps. Whether such constraints are specific to stimulation-induced plasticity is an important question for future research. Weinberger (1998a) notes that the neural feasibility of Hebbian-like learning processes is unclear and that the empirical support for theories invoking Hebbian learning is not as strong as might be supposed (see also Cruikshank \& Weinberger, 1996a; Edeline, 1996, 1999).

Comparisons between specific changes in the response characteristics of map nodes and response characteristics reported in electrophysiological studies are difficult to make, because most reports only describe changes in the responses of individual neurons and the types of changes reported vary considerably within and across studies. Despite this variability, there do not appear to be any reports suggesting that response characteristics in auditory cortical neurons "flatten" before retuning to a new frequency, as we observed in our simulations. Most studies report increases/decreases in the sensitivity of neurons to the conditioned frequency; only minor modifications in previous spectral sensitivities have been reported (see, e.g., Edeline et al., 1990; Edeline \& Weinberger, 1993b; Metherate \& Weinberger, 1990; Ohl \& Scheich, 1996, 1997).

Discrepancies such as those noted above suggest that although self-organizing maps are able to generate global changes in the topographic structure of response properties that are qualitatively similar to those induced by pairing tones with basal forebrain stimulation, the predictive value of such maps may be limited to describing end states of cortical reorganization involving individual tone pips. How do self-organizing maps successfully predict such end states? Presumably, they do so by capturing important processing characteristics of the auditory cortex, including (1) incremental, localized adaptability, (2) processing units that are spatially organized on the basis of similarities between inputs, and (3) competition between processing units. The limitations of self-organizing maps 

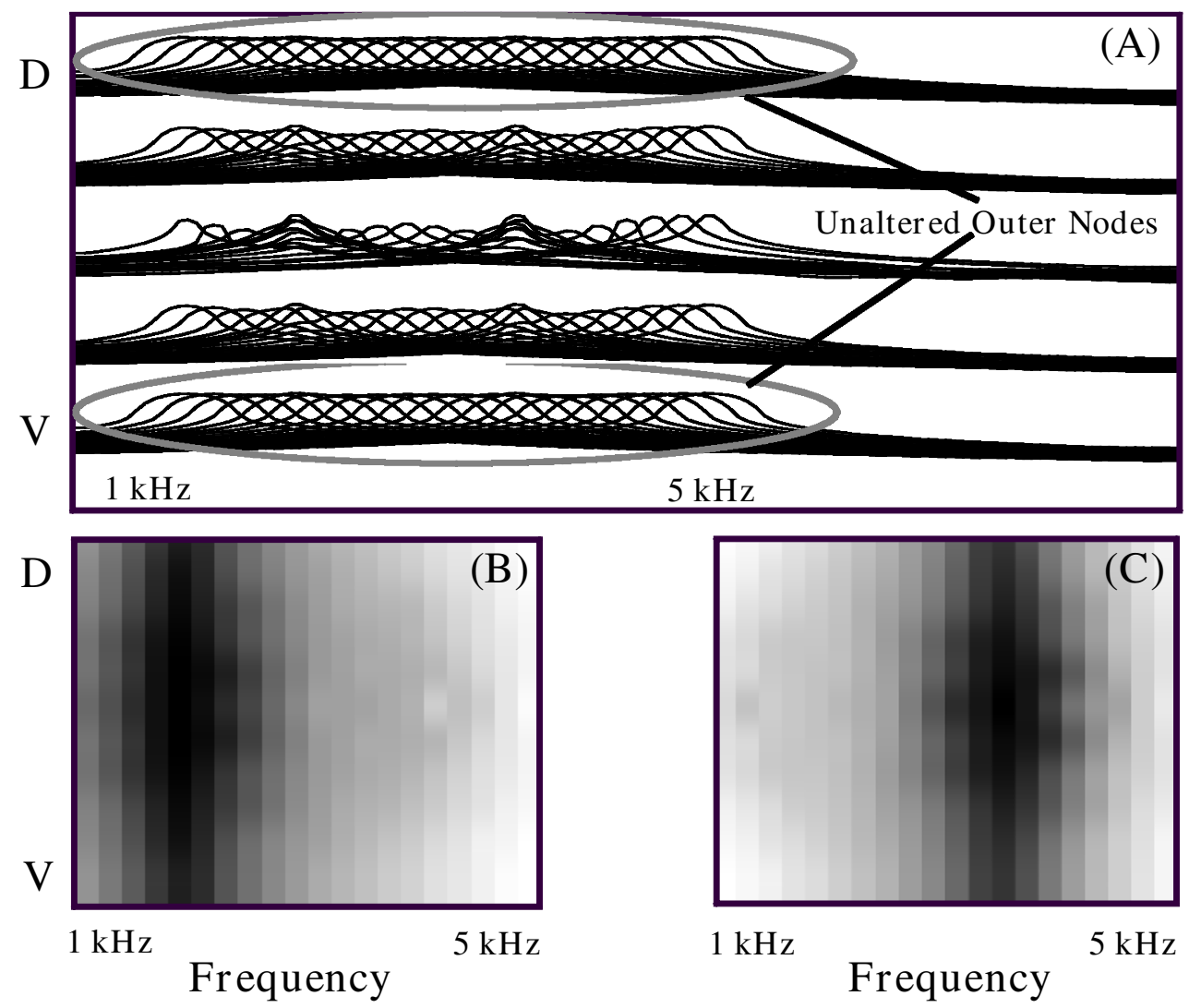

Figure 10. (A) Response properties for a map trained with a $2-\mathrm{kHz}$ tone ( 625 trials) and a 4-kHz tone (625 trials), using a low neighborhood size and a medium learning rate. Changes in response sensitivities of narrowband nodes are minimal, and nodes in dorsal and ventral regions of the map are unaltered. (B) and (C) show that map nodes most sensitive to $2 \mathrm{kHz}(\mathrm{B})$ and $4 \mathrm{kHz}(\mathrm{C})$ have changed little from their initial states.

probably stem from the properties of cortical processing that they do not emulate (e.g., temporal dynamics, speciesspecific predispositions, effects of context, and the order of stimulus presentation). Additional simulations with more advanced self-organizing, map-based architectures can clarify how such properties influence map reorganization.

If the response characteristics of reorganized auditory cortices were measured in sufficient detail, one could better assess the applicability of our simulation results. For example, if basal forebrain stimulation only increases the adaptability of synaptic connections, our model predicts that auditory cortices reorganized by such stimulation should exhibit discrete shifts in response characteristics at the borders of expanded regions of sensitivity. Alternatively, if basal forebrain stimulation mainly increases the excitability of cortical neurons, our model predicts that cortical response characteristics should show a gradual change in sensitivity to the frequency paired with stimulation as distance from the area of greatest sensitivity increases. Recently developed chronic invasive techniques (deCharms, Blake, \& Merzenich, 1999) and noninvasive mapping techniques (Bakin, Kwon, et al., 1996;
Pantev et al., 1999) that allow repeated cortical measurements to be made should provide much needed data regarding the dynamics of auditory cortical reorganization.

The motivation for these simulations was to develop a simple computational framework that would be useful for modeling how neuromodulation affects auditory cortical plasticity. Our model makes specific predictions about how response topography will change as a function of enhanced neural adaptability and excitability in auditory cortical networks. An important line for future research will be to dissociate the effects of different neuromodulators (and neuromodulatory systems) on cortical reorganization. We hypothesize that different neuromodulators differentially affect neural excitability and adaptability, depending on the behavioral context, and that these differential effects can lead to measurable differences in cortical reorganization. For example, if cholinergic modulation increases the excitability of auditory cortical neurons to a greater extent than does dopaminergic modulation, this should lead to predictable differences in the cortical reorganization induced by stimulating neurons in the basal forebrain versus the ventral 
Table 2

Summary of Parameter Effects on Map Reorganization

\begin{tabular}{cll}
\hline \multirow{2}{*}{ Adaptability } & \multicolumn{2}{c}{ Excitability } \\
\cline { 2 - 3 } Low & \multicolumn{1}{c}{ Simulations With One Tone } \\
Little reorganization & \multicolumn{1}{c}{ High } \\
High & Moderate reorganization & Graded changes in sensitivities \\
& Discrete changes in sensitivities & $\begin{array}{l}\text { Massive reorganization } \\
\text { Graded changes in sensitivities }\end{array}$ \\
& & \multicolumn{1}{c}{ Simulations With Two Tones } \\
Low & Little reorganization & Moderate reorganization \\
& & Graded changes in sensitivities \\
& & Bimodally distributed responses \\
& & Symmetry of map shifts \\
High & Moderate reorganization & Massive reorganization \\
& Discrete changes in sensitivities & Graded changes in sensitivities \\
& Bimodally distributed responses & Bimodally distributed responses \\
& Symmetry of map unaffected & Symmetry of map shifts \\
\hline
\end{tabular}

tegmental area (the primary source of dopaminergic neurons projecting to auditory cortex).

Preliminary data suggest that pairing sound with ventral tegmental stimulation does lead to characteristic changes in cortical maps that differ from those associated with basal forebrain stimulation (Bao \& Merzenich, 2000). Pairing tone pips with basal forebrain stimulation in rats causes the regions of the auditory cortex responding to those tone pips to greatly expand (Kilgard \& Merzenich, 1998a), consistent with the reorganization we observed in self-organizing maps with highly excitable nodes. In contrast, pairing tone pips with ventral tegmental stimulation leads to changes that are spatially and spectrally selective (Bao \& Merzenich, 2000), consistent with changes we observed in maps with highly adaptable nodes. Model-based characterizations of differences in the distribution and properties of adapted receptive fields provide a unified framework for quantitatively comparing and contrasting the effects of neuromodulators on cortical plasticity.

One of the major reasons for our interest in understanding how neuromodulation affects auditory cortical reorganization is that most neuromodulatory systems that have been implicated in cortical plasticity have also been implicated in modulating learning and memory processes. For example, the basal forebrain system is believed to be critical in mediating mnemonic and attentional processes (for reviews, see Bartus, Dean, Pontecorvo, \& Flicker, 1985; Dekker, Connor, \& Thal, 1991; Everitt \& Robbins, 1997; Kesner, 1988; Sarter \& Bruno, 1997). Acetylcholine release is enhanced in the initial stages of learning, and the neuromodulatory actions of acetylcholine are often necessary for associative conditioning to occur (Butt, Testylier, \& Dykes, 1997; Jacobs \& Juliano, 1995; Maalouf et al., 1998; Miranda \& Bermudez-Rattoni, 1999; Wellman \& Pelleymounter, 1999). These findings suggest that the role of the nucleus basalis in cortical plasticity is to raise acetylcholine levels when behaviorally relevant events are occur- ring (for reviews, see Ahissar \& Ahissar, 1994; Ashe \& Weinberger, 1991; Metherate \& Weinberger, 1990; Richardson \& Delong, 1991).

In this paper, we focused on simulating the effects of basal forebrain modulation on cortical map reorganization, rather than on simulating receptive field plasticity induced by conditioning. Learning-induced plasticity may involve other modulatory systems in addition to the basal forebrain, and different systems may be involved at different stages of training and/or in different training tasks (Ahissar, Haidarliu, \& Shulz, 1996; Dimyan \& Weinberger, 1999; Sachdev et al., 1998; Stark \& Scheich, 1997; Weinberger, 1998b). Changes in receptive fields can be induced in as few as 5-30 training trials (Bakin \& Weinberger, 1990; Bjordahl et al., 1998; Edeline \& Weinberger, 1993a). Whether such changes reflect topographical reorganization in the auditory cortex is an important question for future research. Our model in its present form is not adequate for simulating several important characteristics of learning-induced cortical plasticity, such as consolidation (Bjordahl et al., 1998; Edeline \& Weinberger, 1993b) and habituation (Condon \& Weinberger, 1991). The roles neuromodulators play in cortical changes induced either by neural processes leading to consolidation or by learning processes, such as habituation and extinction, remain poorly understood. Extension of the model to account for neuromodulatory effects on such processes remains a future line of research.

Simulations with a single tone can potentially be used to model a variety of learning tasks involving cortical plasticity, such as classical fear conditioning, sensory preconditioning, and motor reflex conditioning. Simulations of plasticity-inducing learning tasks that involve either multiple stimuli (e.g., two-tone discrimination or reversal learning; Bakin, South, \& Weinberger, 1996; Bakin \& Weinberger, 1990; Edeline et al., 1990; Edeline \& Weinberger, 1993b; Ohl \& Scheich, 1996) or modulation by other neural regions, such as the hippocampus, would be particularly interesting. The self-organizing map model 
can help identify behavioral tasks in which chemical alteration of neural excitability or adaptability in the auditory cortex might be expected to differentially affect learning. Thus, the model can be useful not only for characterizing neuromodulatory effects on auditory cortical plasticity, but also for predicting how such effects influence learning and memory processes.

\section{REFERENCES}

Аввотт, L., \& SEJNOwSKi, T. (EDS.) (1999). Neural codes and distributed representations: Foundations of neural computation. Cambridge, MA: MIT Press.

Ahissar, E., Abeles, M., Ahissar, M., Haidarliu, S., \& Vaadia, E. (1998). Hebbian-like functional plasticity in the auditory cortex of the behaving monkey. Neuropharmacology, 37, 633-655.

Ahissar, E., \& AhISSAR, M. (1994). Plasticity in auditory cortical circuitry. Current Opinion in Neurobiology, 4, 580-587.

Ahissar, E., Haidarliu, S., \& Shulz, D. E. (1996). Possible involvement of neuromodulatory systems in cortical Hebbian-like plasticity. Journal of Physiology, 90, 353-360.

Ahissar, E., Vaaida, E., Ahissar, M., Bergman, H., Arieli, A., \& ABELES, M. (1992). Dependence of cortical plasticity on correlated activity of single neurons and of behavioral context. Science, 257, 1412-1415.

Aitkin, L. (1990). The auditory cortex. London: Chapman and Hall.

ARbIB, M. A. (ED.) (1998). The handbook of brain theory and neural networks. Cambridge, MA: MIT Press.

Armony, J. L., Servan-Schreiber,D., Cohen, J. D., \& LeDoux, J. E. (1995). An anatomically constrained neural network model of fear conditioning. Behavioral Neuroscience, 109, 246-257.

Armony, J. L., Servan-Schreiber, D., Cohen, \& LeDoux, J. E. (1997). Computational modeling of emotion: Explorations through the anatomy and physiology of fear conditioning. Trends in Cognitive Sciences, 1, 28-34.

Armony, J. L., Servan-Schreiber, D., Romanski, L. M., Cohen, J. D., \& LeDoux, J. E. (1997). Stimulus generalization of fear responses: Effects of auditory cortex lesions in a computational model and in rats. Cerebral Cortex, 7, 157-165.

Ashe, J. H., McKenna, T. M., \& Weinberger, N. M. (1989). Cholinergic modulation of frequency receptive fields in auditory cortex: II. Frequency-specific effects of anticholinesterases provide evidence for a modulatory action of endogenous ACh. Synapse, 4, 44-54.

Ashe, J. H., \& Weinberger, N. M. (1991). Acetylcholine modulation of cellular excitability via muscarinic receptors: Functional plasticity in auditory cortex. In R. T. Richardson (Ed.), Activation to acquisition: Functional aspects of the basal forebrain cholinergic system (pp. 189-246). Boston: Birkhauser.

Bakin, J. S., Kwon, M. C., Masino, S. A., Weinberger, N. M., \& Frostig, R. D. (1996). Suprathreshold auditory cortex activation visualized by intrinsic signal optical imaging. Cerebral Cortex, 6 , 120-130.

BAKIN, J. S., LePAN, B., \& Weinberger, N. M. (1992). Sensitization induced receptive field plasticity in the auditory cortex is independent of CS-modality. Brain Research, 577, 226-235.

Bakin, J. S., South, D. A., \& Weinberger, N. M. (1996). Induction of receptive field plasticity in the auditory cortex of the guinea pig during instrumental avoidance training. Behavioral Neuroscience, 110, 905-913.

BAKIN, J. S., \& WeInberger, N. M. (1990). Classical conditioning induces CS-specific receptive field plasticity in the auditory cortex of the guinea pig. Brain Research, 536, 271-286.

BAKIN, J. S., \& WEINBERGER,N. M. (1996). Induction of a physiological memory in the cerebral cortex by stimulation of the nucleus basalis. Proceedings of the National Academy of Sciences, 93, 11219-11224.

BAo, S., \& Merzenich, M. M. (2000). Auditory cortical reorganization induced by stimulation of ventral tegmental area. Society for Neuroscience Abstracts, 26, 1477.

Barkai, E., Bergman, R. E., Horowitz, G., \& Hasselmo, M. E.
(1994). Modulation of associative memory function in a biophysical simulation of rat piriform cortex. Journal of Neurophysiology, $\mathbf{7 2}$, 659-677.

Bartus, R. T., Dean, R. L., Pontecorvo, M. J., \& Flicker, C. (1985). The cholinergic hypothesis: A historical overview, current perspective, and future directions. In D. S. Olton, E. Gamzu, \& S. Corkin (Eds.), Memory dysfunctions: An integration of animal and human research from preclinical and clinical perspectives (Annals of the New York Academy of Sciences, Vol. 444, pp. 332-358). New York: New York Academy of Sciences.

Baskerville, K. A., Schweitzler,J. B., \& Herron, P. (1997). Effects of cholinergic depletion on experience-dependent plasticity in the cortex of the rat. Neuroscience, 80, 1159-1169.

Bauer, H.-U., Der, R, \& Herrmann, M. (1996). Controlling the magnification factor of self-organizing feature maps. Neural Computation, 8, 757-771.

Benukskova, L., Diamond, M. E., \& Ebner, F. F. (1994). Dynamic synaptic modification threshold: Computational model of experiencedependent plasticity in adult rat barrel cortex. Proceedings of the National Academy of Sciences, 91, 4791-4795.

Bjordahl, T. S., Dimyan, M. A., \& Weinberger, N. M. (1998). Induction of long-term receptive field plasticity in the auditory cortex of the waking guinea pig by stimulation of the nucleus basalis. $\mathrm{Be}$ havioral Neuroscience, 112, 467-479.

Bower, J. M., \& BeEman, D. (1998). The book of GENESIS: Exploring realistic neural models with the GEneral NEural SImulation System. Berlin: Springer-Verlag.

Buonomano, D. V., \& Merzenich, M. M. (1998). Cortical plasticity: From synapses to maps. Annual Review of Neuroscience, 21, 149-186.

Butt, A. E., Testy lier, G., \& Dy Kes, R. W. (1997). Acetylcholine release in rat frontal and somatosensory cortex is enhanced during tactile discrimination learning. Psychobiology, 25, 18-33.

Cansino, S., \& Williamson, S. J. (1997). Neuromagnetic fields reveal cortical plasticity when learning an auditory discrimination task. Brain Research, 764, 53-66.

Condon, C. D., \& Weinberger, N. M. (1991). Habituation produces frequency-specific plasticity of receptive fields in the auditory cortex. Behavioral Neuroscience, 105, 416-430.

Cruikshank, S. J., \& Weinberger, N. M. (1996a). Evidence for the Hebbian hypothesis in experience-dependent physiological plasticity of neocortex: A critical review. Brain Research Reviews, 22, 191-228.

Cruikshank, S. J., \& Weinberger, N. M. (1996b). Receptive-field plasticity in the adult auditory cortex induced by Hebbian covariance. Journal of Neuroscience, 16, 861-875.

deCharms, R. C., Blake, D. T., \& Merzenich, M. M. (1999). A multielectrode implant device for the cerebral cortex. Journal of Neuroscience Methods, 93, 27-35.

deCharms, R. C., \& Merzenich, M. M. (1996). Primary cortical representation of sounds by the coordination of action-potential timing. Nature, 381, 610-613.

deCharms, R. C., \& Zador, A. (2000). Neural representation and the cortical code. Annual Review of Neuroscience, 23, 613-647.

DekKer, A. J., Connor, D. J., \& Thal, L. J. (1991). The role of cholinergic projections from the nucleus basalis in memory. Neuroscience \& Biobehavioral Reviews, 15, 299-317.

Delacour, J. O., Houcine, O., \& Costa, J. C. (1990). Evidence for a cholinergic mechanism of "learned" changes in the responses of barrel field neurons of the awake and undrugged rat. Neuroscience, 34, 1-8.

de Pinho, M., Mazza, M., \& Roque, A. C. (2000). A biologically-plausible computational model of classical conditioning induced reorganization of tonotopic maps in the auditory cortex. Neurocomputing, 32, 685-691.

de Pinho, M., \& Roque-da-Silva, A. C. (1999). A realistic computational model of formation and variability of tonotopic maps in the auditory cortex. Neurocomputing, 26-7, 355-359.

Diamond, D. M., \& Weinberger, N. M. (1984). Physiological plasticity of single neurons in auditory cortex of the cat during acquisition of pupillary responses: II. Secondary field (AII). Behavioral Neuroscience, 98, 189-210.

Diamond, D. M., \& Weinberger,N. M. (1986). Classical conditioning rapidly induces specific changes in frequency receptive fields of sin- 
gle neurons in secondary and ventral ectosylvian auditory cortical fields. Brain Research, 372, 357-360.

Diamond, D. M., \& Weinberger, N. M. (1989). Role of context in the expression of learning-induced plasticity of single neurons in auditory cortex. Behavioral Neuroscience, 103, 471-494.

DiAmond, M. E., \& EbNER, F. F. (1990). Emergence of radial and modular units in neocortex.In B. L. Finlay, G. Innocenti, \& H. Scheich (Eds.), The neocortex: Ontogeny and phylogeny (pp. 159-171). New York: Plenum.

Diamond, M. E., Petersen, R. S., \& Harris, J. A. (1999). Learning through maps: Functional significance of topographic organization in primary sensory cortex. Journal of Neurobiology, 41, 64-68.

Dimyan, M. A., \& Weinberger, N. M. (1999). Basal forebrain stimulation induces discriminative receptive field plasticity in the auditory cortex. Behavioral Neuroscience, 113, 691-702.

Dy Kes, R. W. (1997). Mechanisms controlling neuronal plasticity in somatosensory cortex. Canadian Journal of Physiological Pharmacology, 75, 535-545.

EDELINE, J.-M. (1995). The $\alpha 2$-adrenergic antagonist Idazoxan enhances the frequency selectivity and increases the threshold of auditory cortex neurons. Experimental Brain Research, 107, 221-240.

Edeline, J.-M. (1996). Does Hebbian synaptic plasticity explain learning-induced sensory plasticity in adult mammals? Journal of Physiology, 90, 271-276.

EDELINE, J.-M. (1999). Learning-induced physiological plasticity in thalamo-cortical sensory systems: A critical evaluation of receptive field plasticity, map changes and their potential mechanisms. Progress in Neurobiology, 57, 165-224.

Edeline, J.-M., Hars, B., Maho, C., \& Hennevin, E. (1994). Transient and prolonged facilitation of tone-evoked responses induced by basal forebrain stimulation in the rat auditory cortex. Experimental Brain Research, 97, 373-386.

Edeline, J.-M., Neuenschwander-El Massioui, N., \& Dutrieux, G. (1990). Frequency-specific cellular changes in the auditory system during acquisition and reversal of discriminative conditioning. Psychobiology, 18, 382-393.

Edeline, J.-M., \& Weinberger, N. M. (1993a). Rapid development of learning-induced receptive field plasticity in the auditory cortex. $\mathrm{Be}$ havioral Neuroscience, 107, 539-557.

EdELINE, J.-M., \& WeInBERGER, N. M. (1993b). Receptive field plasticity in the auditory cortex during frequency discrimination training: Selective retuning independent of task difficulty. Behavioral Neuroscience, 107, 82-103.

Ehret, G. (1997). The auditory cortex. Journal of Comparative Physiology A, 181, 547-557.

Elliot, T., \& Shadbolt, N. R. (1998). A model of activity-dependent anatomical inhibitory plasticity applied to the mammalian auditory system. Biological Cybernetics, 78, 455-464.

Erwin, E. Obermayer, K., \& Schulten, K. (1995). Models of orientation and ocular dominance columns in the visual cortex: A critical comparison. Neural Computation, 7, 425-468.

Everitt, B. J., \& Robbins, T. W. (1997). Central cholinergic systems and cognition. Annual Review of Psychology, 48, 649-684.

Fellous, J.-M., \& LinSTER, C. (1998). Computational models of neuromodulation. Neural Computation, 10, 771-805.

Foeller, E., VAter, M., \& Kossl, M. (2000). Influence of GABAergic inhibition on temporal response properties and frequency tuning of auditory cortex neurons in the gerbil. Abstracts of the Association for Research in Otolaryngology, 23, 12.

Fregnac, Y., \& Shulz, D. E. (1999). Activity-dependent regulation of receptive field properties of cat area 17 by supervised Hebbian learning. Journal of Neurobiology, 41, 69-82.

GAO, E., \& Suga, N. (2000). Experience-dependent plasticity in the auditory cortex and the inferior colliculus of bats: Role of the corticofugal system. Proceedings of the National Academy of Sciences, 97, 8081-8086.

Giorgetti, M., Bacciottini, L., Giovannini, M. G., Colivicchi, M. A., Goldfarb, J. O., \& Blandina, P. (2000). Local GABAergic modulation of acetylcholine release from the cortex of freely moving rats. European Journal of Neuroscience, 12, 1941-1948.

Gluck, M. A., \& Myers, C. E. (2001). Gateway to memory: An intro- duction to neural network modeling of the hippocampus and learning. Cambridge, MA: MIT Press.

GonZalez-Lima,F., \& Scheich,H. (1986). Neural substrates for toneconditioned bradycardia demonstrated with 2 deoxyglucose: II. Auditory cortex plasticity. Behavioural Brain Research, 20, 281-293.

GrajsKi, K. A., \& Merzenich, M. M. (1990). Hebb-type dynamics is sufficient to account for the inverse magnification rule in cortical somatotopy. Neural Computation, 2, 71-84.

Gritti, I., Mainville, L., Mancia, M., \& Jones, B. F. (1997). GABAergic and other noncholinergic basal forebrain neurons, together with cholinergic neurons, project to the mesocortex and isocortex in the rat. Journal of Comparative Neurology, 383, 163-177.

Guenther, F. H., \& GJaJA, M. N. (1996). The perceptual magnet effect as an emergent property of neural map formation. Journal of the Acoustical Society of America, 100, 1111-1121.

Hars, B., Maho, C., Edeline, J.-M., \& Hennevin, E. (1993). Basal forebrain stimulation facilitates tone-evoked responses in the auditory cortex of the awake rat. Neuroscience, 56, 61-74.

Hasselmo, M. E. (1995). Neuromodulation and cortical function: Modeling the physiological basis of behavior. Behavioural Brain Research, 67, 1-27.

Jacobs, S. E., Code, R. A., \& Juliano, S. L. (1991). Basal forebrain lesions alter stimulus-evoked metabolic activity in rat somatosensory cortex. Brain Research, 560, 342-345.

JACOBS, S. E., \& Juliano, S. L. (1995). The impact of basal forebrain lesions on the ability of rats to perform a sensory discrimination task involving barrel cortex. Journal of Neuroscience, 14, 697-711.

Jiminez-Capdeville, M. E., Dykes, R. W., \& Myasnikov, A. A. (1997). Differential control of cortical activity by the basal forebrain in rats: A role for both cholinergic and inhibitory influences. Journal of Comparative Neurology, 381, 53-67.

Joublin, F., Spengler, F., Wacquant, S., \& Dinse, H. R. (1996). A columnar model of somatosensory reorganizational plasticity based on Hebbian and non-Hebbian learning rules. Biological Cybernetics, 74, 275-286.

Juliano, S. L., MA, W., \& Eslin, D. (1991). Cholinergic depletion prevents expansion of topographic maps in somatosensory cortex. Proceedings of the National Academy of Sciences, 88, 780-784.

KAAS, J. H. (1996). Plasticity of sensory representations in the auditory and other systems of adult mammals.In R. J. Salvi \& D. Henderson (Eds.), Auditory system plasticity and regeneration (pp. 213-223). New York: Thieme Medical Publishers.

KAAS, J. H. (1997). Introduction: Functional plasticity in adult cortex. Seminars in Neuroscience, 9, 1-1.

KESNER, R. P. (1988). Reevaluation of the contribution of the basal forebrain cholinergic system to memory. Neurobiology of Aging, 9, 609616.

Kilgard, M. P., \& Merzenich, M. M. (1998a). Cortical map reorganization enabled by nucleus basalis activity. Science, 279, 1714-1718.

Kilgard, M. P., \& Merzenich, M. M. (1998b). Plasticity of temporal information processing in primary auditory cortex. Nature Neuroscience, 1, 727-731.

KoHonen, T. (1987). Adaptive, associative, and self-organizing functions in neural computing. Applied Optics, 26, 4910-4918.

KoHonen, T. (1993). Physiological interpretation of the self-organizing mapping algorithm. Neural Networks, 6, 895-905.

KoHONEN, T. (1997). Self-organizing maps. Berlin: Springer-Verlag.

Kraus, N., \& Disterhoft, J. F. (1982). Response plasticity of single neurons in the rabbit auditory association cortex during tone-signaled learning. Brain Research, 246, 205-215.

Maalouf, M., Miasnikov, A. A., \& Dykes, R. W. (1998). Blockade of cholinergic receptors in rat barrel cortex prevents long-term changes in the evoked potentials during sensory preconditioning. Journal of Neurophysiology, 80, 529-545.

Maldonado, P. E., \& Gerstein, G. L. (1996). Reorganization in the auditory cortex of the rat induced by intracortical microstimulation: A multiple single-unit study. Experimental Brain Research, 112, 420-430.

ManunTA, Y., \& Edeline, J.-M. (1997). Effects of noradrenaline on frequency tuning of rat auditory cortex neurons. European Journal of Neuroscience, 9, 833-847.

McKenna, T. M., Ashe, J. H., \& Weinberger, N. M. (1989). Cholin- 
ergic modulation of frequency receptive fields in auditory cortex: I. Frequency-specific effects of muscarinic agonists. Synapse, 4, 30-43.

Mercado, E., III, Myers, C. E., \& Gluck, M. A. (1999a). A computational model of basal forebrain modulation of auditory cortical plasticity. Cognitive Neuroscience Society Annual Meeting Program, 102, 81B.

Mercado, E., III, Myers, C. E., \& Gluck, M. A. (1999b). Plasticity of spectral processing in simulated primary auditory cortex. Society for Neuroscience Abstracts, 29, 391.

Mercado, E., III, Myers, C. E., \& Gluck, M. A. (2000). Modeling auditory cortical processing as an adaptive chirplet transform. Neurocomputing, 32-33, 913-919.

Mercado, E., III, Shohamy, D., Orduña, I., Gluck, M. A., \& MerZENICH, M. M. (2000). Plasticity of spectrotemporal sensitivities in auditory cortex. Journal of the Acoustical Society of America A, 107, 2835.

Merzenich, M. M., \& Schreiner, C. E. (1992). Mammalian auditory cortex: Some comparative observations. In D. B. Webster, R. F. Fay, \& A. N. Popper (Eds.), The evolutionary biology of hearing (pp. 673689). New York: Springer-Verlag.

Metherate, R, \& Ashe, J. H. (1991). Basal forebrain stimulation modifies auditory cortex responsiveness by an action at muscarinic receptors. Brain Research, 559, 163-167.

Metherate, R., \& Ashe, J. H. (1993). Nucleus basalis stimulation facilitates thalamocortical synaptic transmission in the rat auditory cortex. Synapse, 14, 132-143.

Metherate, R., Ashe, J. H., \& Weinberger, N. M. (1990). Acetylcholine modifies neuronal acoustic rate-level functions in guinea pig auditory cortex by an action at muscarinic receptors. Synapse, 6, 364368 .

Metherate, R, Tremblay, N., \& Dykes, R. W. (1987). Acetylcholine permits long-term enhancement of neuronal responsiveness in cat primary somatosensory cortex. Neuroscience, 22, 75-81.

Metherate, R., \& Weinberger, N. M. (1989). Acetylcholine produces stimulus-specific receptive field alterations in cat auditory cortex. Brain Research, 480, 372-377.

Metherate, R. \& Weinberger, N. M. (1990). Cholinergic modulation of responses to single tones produces tone-specific receptive field alterations in cat auditory cortex. Synapse, 14, 133-145.

Meyer-Base, U., \& Scheich, H. (1995). Artificial implementation of auditory neurons: A comparison of biologically motivated models and a new transfer function oriented model. Biological Cybernetics, 77, 123-130.

Miranda, M. I., \& Bermudez-Rattoni, F. (1999). Reversible inactivation of the nucleus basalis magnocellularis induces disruption of cortical acetylcholine release and acquisition, but not retrieval, of aversive memories. Proceedings of the National Academy of Sciences, 96, 6478-6482.

Molchan, S. E., Sunderland, T., McIntosh, A. R, Herscovitch, P., \& Schreurs, B. G. (1994). A functional anatomical study of associative learning in humans. Proceedings of the National Academy of Sciences, 91, 8122-8126.

Morris, J. S., Friston, K. J., \& Dolan, R. J. (1998). Experiencedependent modulation of tonotopicneural responses in human auditory cortex. Proceedings of the Royal Society of London: Series B, 265, 649-657.

Myers, C. E., Ermita, B. R., Harris, K., Hasselmo, M., Solomon, P., \& GLUCK, M. A. (1996). A computational model of cholinergic disruption of septohippocampal activity in classical eyeblink conditioning. Neurobiology of Learning \& Memory, 66, 51-66.

Myers, C. E., Ermita, B. R., Hasselmo, M., \& Gluck, M. A. (1998). Further implications of a computational model of septohippocampal cholinergic modulation in eyeblink conditioning. Psychobiology, 26, $1-20$.

Myers, C. E., Gluck, M. A., \& Granger, R. (1995). Dissociation of hippocampal and entorhinal function in associative learning: A computational approach. Psychobiology, 23, 116-138.

OHL, F. W., \& Scheich, H. (1996). Differential frequency conditioning enhances spectral contrast sensitivity of units in auditory cortex (field AI) of the alert Mongolian gerbil. European Journal of Neuroscience, $\mathbf{8}, 1001-1017$.
Ohl, F. W., \& Scheich, H. (1997). Learning-induced dynamic receptive field changes in primary auditory cortex of the unanaesthetized Mongolian gerbil. Journal of Comparative Physiology A, 181, 685-696.

Palakal, M. J., Murthy, U., Chittajallu, S. K., \& Wong, D. (1995). Tonotopic representation of auditory responses using self-organizing maps. Mathematical \& Computational Modelling, 22, 7-21.

Palakal, M. J., \& Wong, D. (1999). Cortical representation of spatiotemporal pattern of firing evoked by echolocation signals: Population encoding of target features in real time. Journal of the Acoustical Society of America, 106, 479-490.

Pantev, C., \& Lutkenhoner, B. (2000). Magnetoencephalographic studies of functional organization and plasticity of the human auditory cortex. Journal of Clinical Neurophysiology, 17, 130-142.

Pantev, C., Wollbrink, A., Roberts, L. E., Engelien, A., \& LutKENHONER, B. (1999). Short-term plasticity of the human auditory cortex. Brain Research, 842, 192-199.

Piepenbrock, C., \& Obermayer, K. (2000). The effect of intracortical competition on the formation of topographic maps in models of Hebbian learning. Biological Cybernetics, 82, 345-353.

Pitton, J. W., Wang, K., \& JuAng, B.-H. (1996). Time-frequency analysis and auditory modeling for automatic recognition of speech. Proceedings of the IEEE, 84, 1199-1214.

RAUSCHECKER, J. P. (1999). Auditory cortical plasticity: A comparison with other sensory systems. Trends in Neurosciences, 22, 74-80.

Recanzone, G. H., Schreiner, C. E., \& Merzenich, M. M. (1993). Plasticity in the frequency representation of primary auditory cortex following discrimination training in adult owl monkeys. Journal of Neurophysiology, 13, 87-103.

Recanzone, G. H., Schreiner, C. E., Sutter, M. L., Beitel, R. E., \& Merzenich, M. M. (1999). Functional organization of spectral receptive fields in the primary auditory cortex of the owl monkey. Journal of Comparative Physiology A, 185, 493-508.

Richardson, R. T., \& Delong, M. R. (1991). Functional implication of tonic and phasic changes in nucleus basalis neurons. In R. T. Richardson (Ed.), Activation to acquisition: Functional aspects of the basal forebrain cholinergic system (pp. 135-166). Boston: Birkhauser.

Ritter, H., Martinez, T., \& Schulten, K. (1992). Neural computation and self-organizing maps: An introduction. Reading, MA: Addison-Wesley.

Robert, A., \& Eriksson, J. L. (1999). A composite model of the auditory periphery for simulating responses to complex sounds. Journal of the Acoustical Society of America, 106, 1852-1864.

Sachdev, R. N. S., Shao-Ming, L., Wiley, R. G., \& Edner, F. F. (1998). Role of the basal forebrain cholinergic projection in somatosensory cortical plasticity. Journal of Neurophysiology, 79, 3216-3228.

Sanchez-Montanes, M. A., Verschure, P. F., \& Konig, P. (2000). Local and global gating of synaptic plasticity. Neural Computation, 12, 519-529.

SARTER,M., \& BRUnO, J. P. (1997). Cognitive functions of cortical acetylcholine: Toward a unifying hypothesis. Brain Research Reviews, 23, 28-46.

Scheich, H., Stark, H., Zuschratter, W., Ohl, F. W., \& Simonis, C. E. (1997). Some functions of primary auditory cortex in learning and memory formation. In H.-J. Freund, B. A. Sabel, \& O. W. Witte (Eds.), Brain plasticity, Advances in neurology (Vol. 73, pp. 179193). Philadelphia: Lippincott-Raven.

Schreiner, C. E. (1998). Spatial distribution of responses to simple and complex sounds in primary auditory cortex. Audiology \& Neurootology, 3, 104-122.

Schreiner, C. E., Read, H. L., \& Sutter, M. L. (2000). Modular organization of frequency integration in primary auditory cortex. Annual Review of Neuroscience, 23, 501-529.

Shulz, D. E., Sosnik, R., Ego, V., Haidarliu, S., \& Ahissar, E. (2000). A neuronal analogue of state-dependent learning. Nature, 403, 549-553.

Schulze, H., \& Langner, G. (1999). Auditory cortical responses to amplitude modulations with spectra above frequency receptive fields: Evidence for wide spread spectral integration. Journal of Comparative Physiology A, 185, 493-508.

Sirosh, J., \& MiikKulainen, R. (1997). Topographic receptive fields 
and patterned lateral interaction in a self-organizing model of the primary visual cortex. Neural Computation, 9, 577-594.

Stark, H., \& Scheich, H. (1997). Dopaminergic and serotonergic systems are differentially involved in auditory cortex learning: A longterm microdialysis study of metabolites. Journal of Neurochemistry, 68, 691-697.

SugA, N. (1990). Cortical computational maps for auditory imaging. Neural Networks, 3, 3-21.

Suga, N. (1995). Processing of auditory information carried by speciesspecif ic complex sounds. In M. S. Gazzaniga (Ed.), The cognitive neurosciences (pp. 295-313). Cambridge, MA: MIT Press.

Sutton, G. G., III, Reggia, J. A., Armentrout, S. L., \& D'Autrechy, C. L. (1994). Cortical map reorganization as a competitive process. Neural Computation, 6, 1-13.

Swindale, N. V. (1996). The development of topography in the visual cortex: A review of models. Network: Computation in Neural Systems, 7, 161-247.

Swindale, N. V., \& Bauer, H.-U. (1998). Application of Kohonen's self-organizing feature map alg54orithm to cortical maps of orientation and direction preference. Proceedings of the Royal Society of London: Series B, 265, 827-838.

TANAKA, S. (1990). Theory of self-organization of cortical maps: Mathematical framework. Neural Networks, 3, 625-640.

TCHORZ, J., \& Kollmeier, B. (1999). A model of auditory perception as a front end for automatic speech recognition. Journal of the Acoustical Society of America, 106, 2040-2050.

Tremblay, N., Warren, R. A., \& Dy Kes, R. W. (1990). Electrophysiological studies of acetylcholine and the role of the basal forebrain in the somatosensory cortex of the cat. I. Cortical neurons excited by glutamate. Journal of Neurophysiology, 64, 1199-1211.

WAng, J., Caspary, D., \& Salvi, R. J. (2000). GABA-A antagonist causes dramatic expansion of tuning in primary auditory cortex. NeuroReport, 11, 1137-1140.

Wang, K., \& Shamma, S. A. (1995a). Auditory analysis of spectrotemporal information in acoustic signals. IEEE Engineering in Medicine \& Biology, 14, 186-194.

Wang, K., \& Shamma, S. A. (1995b). Spectral shape analysis in the central auditory system. IEEE Transactions on Speech \& Audio Processing, 3, 382-395.

Wang, X., Merzenich, M. M., Beitel, R., \& Schreiner, C. H. (1995). Representation of a species-specific vocalization in the primary auditory cortex of the common marmoset: Temporal and spectral characteristics. Journal of Neurophysiology, 74, 2685-2706.

Webster, H. H., Hanisch, U. K., Dy Kes, R. W., \& Biesold, D. (1991). Basal forebrain lesions with or without reserpine injection inhibit cortical reorganization in a rat hindpaw primary somatosensory cortex following sciatic nerve section. Somatosensory Motor Research, 8, 327-346.

Webster, H. H., Rasmusson, D. D., Dykes, R. W., Schliebs, R., Schober, W., Bruckner, G., \& Biesold, D. (1991). Long-term enhancement of evoked potentials in raccoon somatosensory cortex following co-activation of the nucleus basalis of Meynert complex and cutaneous receptors. Brain Research, 545, 292-296.
Weinberger, N. M. (1993). Learning-induced changes of auditory receptive fields. Current Opinion in Neurobiology, 3, 570-577.

WeINBERGER, N. M. (1995). Dynamic regulation of receptive fields and maps in the adult sensory cortex. Annual Review of Neuroscience, $\mathbf{1 8}$, 129-158.

WEINBERGER, N. M. (1997). Learning-induced receptive field plasticity in the primary auditory cortex. Seminars in Neuroscience, 9, 59-67.

WeINBERgER, N. M. (1998a). Physiological memory in primary auditory cortex: Characteristics and mechanisms. Neurobiology of Learning \& Memory, 70, 226-251.

Weinberger, N. M. (1998b). Tuning the brain by learning and by stimulation of the nucleus basalis. Trends in Cognitive Sciences, 2, 271-273.

Weinberger, N. M., Ashe, J. H., Metherate, R., McKenna, T. M., DiAMOND, D. M., \& BAKIN, J. (1990a). Retuning auditory cortex by learning: A preliminary model of receptive field plasticity. Concepts in Neuroscience, 1, 91-132.

Weinberger, N. M., Ashe, J. H., Metherate, R., McKenna, T. M., Diamond, D. M., Bakin, J. S., Lennartz, R. C., \& Cassady, J. M. (1990b). Neural adaptive information processing: A preliminary model of receptive field plasticity in auditory cortex during Pavlovian conditioning. In M. Gabriel \& J. Moore (Eds.), Neurocomputation and learning: Foundations of adaptive networks (pp. 91-138). Cambridge, MA: MIT Press.

WEINBERGER, N. M., \& BAKIN, J. S. (1998). Learning-induced physiological memory in adult primary auditory cortex: Receptive field plasticity, model, and mechanisms. Audiology \& Neuro-Otology, 3, 145167.

Weinberger,N. M., \& Diamond, D. (1987). Physiological plasticity in auditory cortex: Rapid induction by learning. Progress in Neurobiology, 29, 1-55.

Weinberger, N. M., Hopkins, W., \& Diamond, D. M. (1984). Physiological plasticity of single neurons in auditory cortex of the cat during acquisition of pupillary responses: I. Primary field (AI). Behavioral Neuroscience, 98, 171-188.

WEINBERGER,N. M., JAVID, R, \& LEPAN, B. (1993). Long-term retention of learning-induced receptive-field plasticity in the auditory cortex. Proceedings of the National Academy of Sciences, 90, 2394-2398.

Wellman, C. L., \& Pelleymounter, M. A. (1999). Differential effects of nucleus basalis lesions in young adult and aging rats. Neurobiology of Aging, 20, 381-393.

Zaborszky, L., Pang, K., Somogyi, J., Nadasdy, Z., \& Kallo, I. (1999). The basal forebrain corticopetal system revisited. In J. F. McGinty (Ed.), Advancing from the ventral striatum to the extended amygdala: Implicationsfor neuropsychiatry and drug abuse. (Annals of the New York Academy of Sciences, Vol. 877, pp. 339-367). New York: New York Academy of Sciences.

Zoli, M., Torri, C., Jansson, A., Zini, I., Fuze, K., \& Agnati, L. F. (1998). The emergence of the volume transmission concept. Brain Research Reviews, 26, 136-147.

(Manuscript received May 10, 2000; revision accepted for publication September 11, 2000.) 